Pacific Journal of Mathematics

CONGRUENCE LATTICES OF ALGEBRAS OF FIXED 


\title{
CONGRUENCE LATTICES OF ALGEBRAS OF FIXED SIMILARITY TYPE, II
}

\author{
WILliam A. LAMPE
}

A celebrated theorem of G. Grätzer and E. T. Schmidt shows that every algebraic lattice can be represented as the congruence lattice of some universal algebra. That result naturally provokes questions concerning possible refinements. This paper provides sufficient conditions for an algebraic lattice to be representable as the congruence lattice of a groupoid.

Part I, [5], showed that the subspace lattice of each infinite dimensional vector space over any uncountable field is not the congruence lattice of any algebra of countable similarity type. It also presented some necessary conditions for an algebraic lattice to be representable as the congruence lattice of an algebra of countable similarity type.

Suppose $L$ is an algebraic lattice. We shall say that $L$ is a pinched lattice iff there exists a set $I$ of compact elements of $L$ such that $\vee I=1$ and such that each compact element of $L$ is comparable to every element of $I$. Each algebraic lattice with a compact unit element is a pinched lattice. So are ordinal sums of such lattices and certain homomorphic images of such sums.

The principal result of this paper is

THEOREM 1. $L$ is isomorphic to the congruence lattice of a groupoid if $L$ is isomorphic to one of the following:

(i) a pinched lattice;

(ii) the lattice of ideals of a distributive lattice;

(iii) a direct product of lattices satisfying (i) or (ii).

In his 1980 paper [24], E. T. Schmidt shows that the ideal lattice of any distributive lattice can be represented as the congruence lattice of a lattice. From the lattice theory point of view this is a vast improvement over the the appropriate part of Theorem 1 . Also, using McKenzie's type reduction theorem (see [17]), one obtains as a corollary that any such lattice has a representation in similarity type $\langle 2,1\rangle$. As yet, there is no reduction theorem which reduces finite type to type $\langle 2\rangle$. Theorem 1 provides representations in the latter type.

Theorem 1 was announced in 1977 lectures in Budapest, at the 
Esztergom Colloquium and in [17]. The latter includes a survey of this field.

By Theorem 1 we see that the class of lattices isomorphic to congruence lattices of groupoids includes all finite lattices, all chains, and all projective planes. Also, each algebraic lattice $L$ is a retract of an element in this class (see Figure 1).

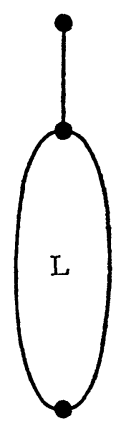

Figure 1

The above theorem was discovered before the results of Part I. In fact, the failure of the author's attempts to improve Theorem 1 led to Part I.

$\S 2$ of the paper contains preliminaries. $\S 3$ is devoted to part (i). The proof of (ii) is in $\S 4 . ~ \S 5$ contains a generalization of (iii). It provides sufficient conditions so that the congruence lattice of a direct sum of algebras is the direct product of the congruence lattices of the algebras. Theorem 1 is a representation theorem. $\S 6$ contains a nonrepresentation theorem (Theorem 5) and concluding remarks.

ACKNOWLEDGMENTS. The author acknowledges helpful discussions with Croy Pitzer and Jiri Sichler. Evelyn Nelson's and Walter Taylor's influence and comments have made the paper less unreadable than it might have been. The research for an early version of this paper [16] was supported by the National Science Foundation under grant GP-37501 and that version was written while the author was a member of the Institute for Advanced Study in 1974-75.

2. Preliminaries. The various lemmas in this section are either well known or trivial. So no proofs are included.

Generally, the terminology will be as in Grätzer's book [8], but the notations will differ somewhat. For example, we will use boldface letters, such as $\boldsymbol{A}$, to denote algebras, while letters such as $A$ will denote the underlying set of an algebra. Suppose $\Theta$ is an equivalence relation. $a / \Theta$ will denote the $\Theta$ class to which $a$ belongs. Both $a \equiv b(\Theta)$ and $a \Theta b$ will be used to denote $\langle a, b\rangle \in \Theta$. For a subset 
$S$ of the domain of $\Theta,\left.\Theta\right|_{S}$ will denote $\Theta \cap(S \times S)$. $\quad \Delta$ is the diagonal or equality relation. Dmn and Rng are used as abbreviations for domain and range, respectively. For example, $\operatorname{Dmn}(\cdot, \boldsymbol{A})$ will mean the domain of the partial operation . in the partial algebra $\boldsymbol{A}$. Con $\boldsymbol{A}$ and $\operatorname{Con}(\boldsymbol{A})$ will both variously denote the set and lattice of congruence relations of $A$.

Suppose $A$ is a set and $C$ is some set of subsets of $A$. For $S \subseteq A$ we set

$$
[S]_{C}=\bigcap(X \in C: S \subseteq X)
$$

provided this intersection is an element of $C$. Otherwise, $[S]_{C}$ is undefined. We call $[S]_{C}$ the $C$-closure of $S$. As usual, $[a]_{C}$ abbreviates $[\{a\}]_{C}$. Obviously, when it exists, $[S]_{C}$ is the $X \in C$ satisfying: (i) $S \subseteq X \in C$; (ii) $S \subseteq Y \in C$ implies $X \subseteq Y$. Recall that $C$ is a closure system iff $[S]_{C}$ exists for each $S \subseteq A . \quad C$ is an algebraic closure system iff $C$ is a closure system and the union of any up-directed subset of $C$ is also an element of $C$. Suppose that $C$ is a collection of subsets of $A \times A$, each of which is an equivalence relation on $A$. Then, for $a, b \in A$ and $p \in A \times A$ we also use $\Theta_{c}(a, b)$ to denote $[\langle a, b\rangle]_{c}$ and $\Theta_{c}(p)$ to denote $[p]_{c}$. Similarly $\Theta_{c}(S)=[S]_{C}$ for $S \subseteq A \times A$. Also, for $a, b, c \in A, \Theta_{C}(a, b, c)=\Theta_{C}(\{\langle a, b\rangle,\langle b, c\rangle\})=[\{\langle a, b\rangle,\langle b, c\rangle\}]_{C}$, etc. If $C=\operatorname{Con} A$, we will, as usual, use $\Theta(a, b)$ instead of $\Theta_{\mathrm{Con} A}(a, b)$. A principal congruence is one of the form $\Theta(a, b)$.

Definition. Suppose $C$ is a collection of subsets of the set $A$. $C$ is a basis iff the $C$-closure of each finite subset of $A$ exists and $C=\left\{[F]_{C}: F\right.$ is a finite subset of $\left.A\right\}$.

Proposition 1. Given a basis $B$, then $C=\{X: X$ is the union of an up-directed subset of $B\}$ is an algebraic closure system. Moreover, given any algebraic closure system $C$, the set $B=\left\{[F]_{C}: F\right.$ is a finite subset of $\cup C\}$ is a basis, and $C$ is the set of unions of up-directed subsets of $B$.

Lemma 2. Suppose $C$ is a collection of subsets of the set $A . \quad C$ is a basis iff the following hold:

(i) $\langle C$; $\leqq\rangle$ is a join semilattice with zero;

(ii) $[a]_{C}$ exists for every $a \in A$;

(iii) $\left\{[a]_{C}: a \in A\right\}$ generates $C$ as a join semilattice.

Proposition 3. Suppose $B$ is a basis on $A$ and $X \subseteq B$. If in $\langle B ; \subseteq\rangle$ the meet (or infimum) of $X$ exists, then $\Lambda X=\bigcap X$.

Lemma 4. Suppose $\boldsymbol{A}$ is a partial algebra, $\Phi \in \operatorname{Con} \boldsymbol{A}, B \cong \operatorname{Con} \boldsymbol{A}$, 
$B$ is a basis on $A \times A$. If $\Phi \subseteq \Theta$ for each $\Theta \in B$, then $B / \Phi=$ $\{\Theta / \Phi: \Theta \in B\}$ is a basis, and $\langle B ; \subseteq\rangle$ is isomorphic to $\langle B / \Phi: \subseteq\rangle$.

Observe that under the above hypotheses, $x \Phi x^{\prime}$ and $y \Phi y^{\prime}$ imply $\Theta_{B}(x, y)=\Theta_{B}\left(x^{\prime}, y^{\prime}\right)$. Whence $\Theta_{B / \Phi}(x / \Phi, y / \Phi)=\left(\Theta_{B}(x, y)\right) / \Phi$. Lemma 4 follows from Lemma 2 .

In the situation under discussion in the preceding paragraph, the relations $\Theta_{B / \phi}(x / \Phi, y / \Phi)$ and $\Theta_{B}(x, y)$ are completely unambiguous, even though there may be some ambiguity as to the pair $\langle x, y\rangle$. At certain parts of the proofs there will be such $x, x^{\prime}, y, y^{\prime}$ where $\Theta_{B}(x, y)$ and $\Theta_{B}\left(x^{\prime}, y^{\prime}\right)$ will have different "natural descriptions." This situation can create an apparent, but spurious, ambiguity in the meaning of $\Theta_{B / \Phi}(x / \Phi, y / \Phi)$.

Suppose $C$ is a basis on $A \times A$ consisting of equivalence relations on $A$ and $D \subseteq A$. Then we say " $x$ is the closest thing to $y$ in $D$, modulo $C^{\prime \prime}$ and we write

\section{$x \operatorname{CLS} y($ in $D, \bmod C)$}

iff the following hold:

(i) $x \in D$;

(ii) $\theta_{C}(x, y) \subseteq \theta_{C}(z, y)$ for every $z \in D$;

(iii) $x=y$ if $y \in D$.

A partial pointed groupoid is a partial algebra $\langle A, \cdot, 0\rangle$ in which $\cdot$ is a binary partial operation and 0 is a nullary operation and 0.0 is defined and equals 0 . A pointed groupoid is a partial pointed groupoid $\langle A, \cdot, 0\rangle$ in which $\operatorname{Dmn}(\cdot)=A \times A$. More generally, $\boldsymbol{A}$ is a pointed algebra iff $\boldsymbol{A}$ is an algebra and 0 is a nullary operation of $\boldsymbol{A}$ and $\{0\}$ is a subalgebra of $\boldsymbol{A}$.

Suppose $A$ and $B$ are sets, $A \cong B, \Theta$ is an equivalence relation on $A$, and $\Phi$ is an equivalence relation on $B$. $\Phi$ is an extension of $\Theta$ iff $\Theta=\left.\Phi\right|_{A}=\Phi \cap(A \times A)$.

Suppose $\boldsymbol{A}=\langle A, \cdot, 0\rangle$ is a partial pointed groupoid. The set $A[\cdot]$ is formed by adding to $A$ a new point for each $\langle x, y\rangle$ such that $x \cdot y$ is undefined in $\boldsymbol{A}$. We intend this new point to be the value of $x \cdot y$. One obtains a partial pointed groupoid $A[\cdot]=\langle A[\cdot], \cdot, 0\rangle$, in which $\operatorname{Dom}(\cdot, \boldsymbol{A}[\cdot])=A^{2}$, called $\boldsymbol{A}$ freely extended by $\cdot$. Note that if $x \cdot y=u \cdot v \in A[\cdot]-A$, then $x=u$ and $y=v . \quad A[\cdot]$ is an extension of $\boldsymbol{A}$. Set $\boldsymbol{A}[\cdot]_{0}=\left\langle A[\cdot]_{0}, \cdot, 0\right\rangle=\boldsymbol{A}$. Set $A[\cdot]_{n+1}=\left\langle A[\cdot]_{n+1}, \cdot, 0\right\rangle=$ $\left(\boldsymbol{A}[\cdot]_{n}\right)[\cdot]$. The pointed groupoid freely generated by $\boldsymbol{A}$ is $\boldsymbol{F r}(\boldsymbol{A})=$ $\langle\boldsymbol{F r}(\boldsymbol{A}), \cdot, 0\rangle=\left\langle\mathbf{U}\left(A[\cdot]_{n}: n=0,1, \cdots\right), \cdot, 0\right\rangle . \quad \boldsymbol{F r}(\boldsymbol{A})$ is a pointed groupoid (i.e., · is fully defined), and $\operatorname{Fr}(\boldsymbol{A})$ satisfies an appropriate universal mapping property. Note that the subalgebra generated by $A$ in $\operatorname{Fr}(A)$ is $\operatorname{Fr}(A)$. 
Lemma 5. Suppose $\boldsymbol{A}=\langle A, \cdot, 0\rangle$ is a partial pointed groupoid and $\Theta \in \operatorname{Con}(\boldsymbol{A}) . \quad \Theta$ has an extension to a congruence relation of $A[\cdot]$, and for its smallest extension, $\Theta[\cdot]$, the following hold:

(i) if $a, b \in A$, then $a \equiv b(\Theta[\cdot])$ iff $a \equiv b(\Theta)$;

(ii) if $a \in A$ and $b=u \cdot v \notin A$. then $a \equiv b(\Theta[\cdot])$ iff there exists $\langle r, s\rangle \in \operatorname{Dom}(\cdot, \boldsymbol{A})$ such that $a \equiv r \cdot s(\Theta), r \equiv u(\Theta)$ and $s \equiv v(\Theta)$;

(iii) if $a=x \cdot y \notin A$ and $b=u \cdot v \notin A$, then $a \equiv b(\Theta[\cdot])$ iff one of the following holds:

(iii $\left.{ }_{1}\right) \quad x \equiv u(\Theta)$ and $y \equiv v(\Theta)$;

$\left(\mathrm{iii}_{2}\right)$ there exist $\langle p, q\rangle,\langle r, s\rangle \in \operatorname{Dom}(\cdot, \boldsymbol{A})$ such that $x \equiv p(\Theta), y \equiv$ $q(\Theta), p \cdot q \equiv r \cdot s(\Theta), r \equiv u(\Theta)$, and $s \equiv v(\Theta)$.

This lemma is simply a specialization of Lemma 3, p. 92 of [8].

Set $\Theta[\cdot]_{0}=\Theta$ and $\Theta[\cdot]_{n+1}=\left(\Theta[\cdot]_{n}\right)[\cdot]$ and $\operatorname{Fr}(\Theta)=\mathbf{U}\left(\Theta[\cdot]_{n}: n=\right.$ $0,1, \cdots)$.

Lemma 6. Suppose $\boldsymbol{A}=\langle A ; \cdot, 0\rangle$ is a partial pointed groupoid and $\Theta \in \operatorname{Con}(\boldsymbol{A})$. Then $\operatorname{Fr}(\Theta)$ is an extension of $\Theta$, and it is the smallest extension of $\Theta$ to a congruence relation of $\boldsymbol{F r}(\boldsymbol{A})$, the groupoid freely generated by $\boldsymbol{A}$.

Suppose $L$ is a lattice of equivalence relations on some set. $L$ is a type-3 partition lattice, or $L$ has type-3 joins, iff $x \vee y=x \circ y \circ x \circ y$ for any $x, y \in L$, where $\circ$ denotes relation composition. $L$ is type-2 iff every $x \vee y=x \circ y \circ x$.

Suppose $L$ is an algebraic lattice. $C(L)$ denotes the set or semilattice of compact elements of $L$.

We will use $x y$ to stand for $x \cdot y$ and $A b$ or $A \cdot b$ for $\{a b: a \in A\}=$ $\{a \cdot b: b \in A\}$, etc.

3. Pinched lattices. Suppose $\alpha$ is an ordinal and $\left(L_{\beta}: \beta<\alpha\right)$ is a family of partially ordered sets. The ordinal sum of $\left(L_{\beta}: \beta<\alpha\right)$, $O \Sigma\left(L_{\beta}: \beta<\alpha\right)$, is a poset on the disjoint union of the family $\left(L_{\beta}: \beta<\alpha\right)$ with the ordering defined by $x \leqq y$ iff $x \in L_{\beta}$ and $y \in L_{\gamma}$ and $\beta<\gamma$ or else $\beta=\gamma$ and $x \leqq y$ in $L_{\beta}$. Suppose each $L_{\beta}$ has a greatest element $1_{\beta}$ and a least element $0_{\beta}$. The collapsed ordinal sum of the family $\left(L_{\beta}: \beta<\alpha\right)-C O \Sigma\left(L_{\beta}: \beta<\alpha\right)$-is the maximal homomorphic image of $O \Sigma\left(L_{\beta}: \beta<\alpha\right)$ satisfying $1_{\beta}=0_{\beta+1}$ for every $\beta$ such that $\beta+1<\alpha$.

Proposition 1. If $L$ is a pinched lattice, then there is a set $I$ of compact element of $L$ such that $I$ is well ordered by the ordering of $L$ and such that $\bigvee I=1$ and such that each compact element of $L$ is comparable to every element $I$. 
Proposition 2. $L$ is a pinched lattice iff one of the following holds:

(a) $L$ is an algebraic lattice whose unit element is compact;

(b) $L$ is isomorphic to an ordinal sum $M+\{1\}$ and $M$ is a collapsed ordinal sum of algebraic lattices each of which has a compact unit element.

In this section we shall show that each pinched lattice can be represented as the congruence lattice of a groupoid. The construction will involve transfinite recursions. The proofs will involve transfinite induction. The induction hypothesis will include the following list of conditions. In that list and in what follows $V$ means the join in $\langle H$; $\leqq$ and not the join in Con $A$.

(\#) (A) $\boldsymbol{A}$ is a partial pointed groupoid.

(B) $H \subseteq \operatorname{Con}(\boldsymbol{A})$ and $\Delta \in H$ and $\rho: A \rightarrow H$.

(C) $H$ is a basis.

(D) $\operatorname{Rng}(\rho)$ is a well ordered cofinal subset of $\langle H$; $\cong\rangle$.

(E) For every $a \in A$ and $\Phi \in H$ it is the case that $\rho(\alpha)$ and $\Phi$ are comparable in $\langle H ; \cong\rangle$.

(F) For every $a \in A$ we have $\rho(a)=\Lambda\{\rho(b): a \equiv 0(\rho(b))\}$.

(G) There is a $D \leqq A$ such that $\varnothing \neq D \times D=\operatorname{Dmn}(\cdot, A)$.

(H) For every $a \in A$ there is a $c \in D$ satisfying $c$ CLS $a$ (in $D$, $\bmod H)$.

(I) For every $a, b \in A$ there are $c, d \in D$ satisfying $\Theta_{H}(a, b) \supseteqq$ $\Theta_{H}(c, d)$ such that $a$ and $c$ satisfy the condition in $\left.H\right)$ and such that $b$ and $d$ also satisfy this condition.

(J) For every $u, v, x, y \in D$ we have either $\Theta_{H}(u x, v y)=\Theta_{H}(u, v) \bigvee$ $\Theta_{H}(x, y)$ or $\Theta_{H}(x, y)=\rho(u, v, x, y)$, where $\rho(u, v, x, y)$ is an abbreviation for $\rho(u) \vee \rho(v) \vee \rho(x) \vee \rho(y)$.

(K) For every $\Theta \in$ Con $A, \Theta$ contains the $H$-closure of each of its finite subsets iff $\Theta$ contains the $H$-closure of each of its elements.

Let $u, r, x, y \in A$. We set $\Phi(u x, v y)=\Theta_{H}(u, v) \vee \Theta_{H}(x, y)$. Note that $\Phi(u x, v y)$ is defined even if $u x$ or $v y$ is not. We set $\Psi(u x, v y)=$ $\Theta_{H}(u, q) \vee \Theta_{H}(x, s) \vee \Theta_{H}(q s, r t) \vee \Theta_{H}(r, v) \vee \Theta_{H}(t, y)$, where $q$ CLS $u$ (in $D, \bmod H)$ and $r$ CLS $v($ in $D, \bmod H)$ and $s$ CLS $x($ in $D, \bmod H)$ and $t$ CLS $y$ (in $D, \bmod H$ ). Suppose $\langle A, H, \rho\rangle$ satisfies (\#) and $\Theta \in H$. Note that $\Psi(u x, v y)$ is independent of the choice of $q, r, s, t$. It follows from Lemma 2.5 that if $u x \in A[\cdot]-A$ and $v y \in A[\cdot]-A$, then $u x \equiv v y(\Theta[\cdot])$ iff $\Phi(u x, v y) \leqq \Theta$ or $\Psi(u v, x y) \leqq \Theta$.

Lemma 3. Suppose $\langle A, H, \rho\rangle$ satisfies (\#). Then, for every $x, y$, $u, v$ there are $q, r, s, t$ as above also satisfying $\Theta_{H}(u, v) \supseteqq \Theta_{H}(q, r)$ and $\Theta_{H}(x, y) \supseteqq \Theta_{H}(s, t)$. Moreover, $\Psi(u x, v y)$ exists and: 
(i ) if $\Theta_{H}(q s, r t)=\Theta_{H}(q, r) \vee \Theta_{H}(s, t)$, then $\Phi(u x, v y) \subseteq \Psi(u x, v y)$;

(ii) if $\Theta_{H}(q s, r t) \neq \Theta_{H}(q, r) \vee \Theta_{H}(s, t)$ and $\Theta_{H}(u, q) \vee \Theta_{H}(x, s) \vee$ $\Theta_{H}(r, v) \vee \Theta_{H}(t, y) \supseteqq \rho(q, r, s, t)$, then $\Phi(u, v y) \leqq \Psi(u x, v y)$;

(iii) if $\Theta_{H}(q s, r t) \neq \Theta_{H}(q, r) \vee \Theta_{H}(s, t)$ and $\Theta_{H}(u, q) \vee \Theta_{H}(x, s) \vee$ $\Theta_{H}(r, v) \vee \Theta_{H}(t, y) \subseteq \rho(q, r, s, t)$, then $\Psi(u x, v y) \subseteq \Phi(u x, v y)$ and $\Theta_{H}(x, y)=$ $\rho(u, v, x, y)$

(iv) $\Phi(u x, v y)$ and $\Psi(u x, v y)$ are comparable.

Proof of Lemma 3. By (H) and (I) of (\#) such $q, r, s, t$ exist, and so $\Psi(u x, v y)$ exists. For (iv), keep in mind that $\Psi(u x, v y)$ is independent of the choice of $q, r, s, t$. The rest now follows easily from $(\mathrm{D}),(\mathrm{E}),(\mathrm{F})$ and $(\mathrm{J})$ of $(\sharp)$ and the following observations. In (ii), we clearly have $\Phi(u x, v y) \leqq \Theta_{H}(u, q) \vee \Theta_{H}(x, s) \vee \Theta_{H}(r, v) \vee \Theta_{H}(t, y)$. In (iii), we have $\rho(q, r, s, t) \supseteqq \Psi(u x, v y)$ and $\Theta_{H}(x, y) \supseteqq \Theta_{H}(s, t)=$ $\rho(q, r, s, t) \supseteqq \Phi(u x, v y) \supseteqq \Theta_{H}(x, y)$. Since $\Theta_{H}(x, y)=\rho(q, r, s, t)$, by (D) and $(\mathrm{F})$ of (\#), $\Theta_{H}(x, y)=\rho(x, y)$. Moreover, $\rho(x, y)=\rho(x, y, u, v)=$ $\rho(q, r, s, t)$.

Definition. Suppose $\langle\boldsymbol{A}, H, \rho\rangle$ satisfies (\#). $\left\langle\boldsymbol{A}^{*}, H^{*}, \rho^{*}\right\rangle$ is an extension of $\langle A, H, \rho\rangle$ iff the following hold:

(i) $\boldsymbol{A}^{*}$ is an extension of $\boldsymbol{A}$;

(ii) $A \times A \subseteq \operatorname{Dmn}\left(\cdot, A^{*}\right)$;

(iii) $[\Theta]_{H^{*}} \cap(A \times A)=\Theta$ for any $\Theta \in H$;

(iv) $H^{*}=\left\{[\Theta]_{H^{*}}: \Theta \in H\right\}$;

(v) For each $a \in A$, we have $\rho^{*}(a)=[\rho(a)]_{H^{*}}$;

(vi) $\operatorname{Rng}\left(\rho^{*}\right)=\left\{[\Theta]_{H^{*}}: \Theta \in \operatorname{Rng} \rho\right\}$;

(vii) $\left\langle\boldsymbol{A}^{*}, H^{*}, \rho^{*}\right\rangle$ also satisfies (\#).

Note that it is implicit in (iii) of this definition that $[\Theta]_{H^{*}}$ is required to exist for each $\Theta \in H$.

Next we state the principal lemmas for this section.

Recall that $C(L)$ is the set or semilattice of compact elements of $L$.

Lemma 4. If $L$ is a pinched lattice, then there is a $\langle\boldsymbol{B}, H, \rho\rangle$ satisfying (甘) with $C(L) \cong\langle H$; $\leqq$.

Set $H[\cdot]=\{\Theta[\cdot]: \Theta \in H\}$. We define $\rho[\cdot]$ by $\rho[\cdot](a)=\rho(a)[\cdot]$ if $a \in A$ and $\rho[\cdot](a b)=(\Lambda\{\rho(c): a b \equiv 0(\rho(c)[\cdot])\})[\cdot]$.

Lemma 5. If $\langle A, H, \rho\rangle$ satisfies (\#), then $H[\cdot]$ is a basis and $\langle\boldsymbol{A}[\cdot], H[\cdot], \rho[\cdot]\rangle$ is an extension of $\langle\boldsymbol{A}, H, \rho\rangle$.

Lemma 6. Suppose: 
(i) $\alpha$ is a limit ordinal;

(ii) for any $\beta\left\langle\gamma\left\langle\alpha\left\langle\boldsymbol{A}_{\gamma}, H_{\gamma}, \rho_{\gamma}\right\rangle\right.\right.$ is an extension of $\left\langle\boldsymbol{A}_{\beta}, H_{\beta}, \rho_{\beta}\right\rangle$;

(iii) $\boldsymbol{A}_{\alpha}=\left\langle A_{\alpha}, \cdot, 0\right\rangle=\left\langle\mathbf{U}\left(A_{\beta}: \beta\langle\alpha), \cdot, 0\right\rangle\right.$;

(iv) for each $\Theta \in H_{0}$ we have $\Theta_{\alpha}=\mathrm{U}\left([\Theta]_{H_{\beta}}: \beta<\alpha\right)$ and $H_{\alpha}=$ $\left\{\Theta_{\alpha}: \Theta \in H_{0}\right\}$

(v) for each $a \in A_{\alpha}$ we have $\rho_{\alpha}(a)=\cup\left(\rho_{\beta}(a): \beta<\alpha\right.$ and $\left.a \in A_{\beta}\right)$. Then $\boldsymbol{A}_{\alpha}$ is a pointed groupoid and $\left\langle\boldsymbol{A}_{\alpha}, H_{\alpha}, \rho_{\alpha}\right\rangle$ is an extension of $\left\langle\boldsymbol{A}_{\beta}, H_{\beta}, \rho_{\beta}\right\rangle$ for all $\beta<\alpha$.

Suppose $\langle\boldsymbol{A}, H, \rho\rangle$ satisfies (\#). Set $\left\langle\boldsymbol{A}_{0}, H_{0}, \rho_{0}\right\rangle=\langle\boldsymbol{A}, H, \rho\rangle$ and set $\left\langle\boldsymbol{A}_{n+1}, H_{n+1}, \rho_{n+1}\right\rangle=\left\langle\boldsymbol{A}_{n}[\cdot], H_{n}[\cdot], \rho_{n}[\cdot]\right\rangle$. Then $\left\langle\boldsymbol{A}_{\omega}, H_{\omega}, \rho_{\omega}\right\rangle=$ $\left\langle\boldsymbol{F r}(\boldsymbol{A}),\{\boldsymbol{F r}(\Theta): \Theta \in H\}, \rho_{\omega}\right\rangle$. So we set $\boldsymbol{F r}(H)=H_{\omega}$ and $\boldsymbol{F r}(\rho)=\rho_{\omega}$.

Lemma 7. If $\langle\boldsymbol{A}, H, \rho\rangle$ satisfies (\#), then $\langle\boldsymbol{F r}(\boldsymbol{A}), \boldsymbol{F r}(H), \boldsymbol{F r}(\rho)\rangle$ is an extension of $\langle\boldsymbol{A}, H, \rho\rangle$.

Suppose that $\langle\boldsymbol{A}, H, \rho\rangle$ satisfies (\#) and $\operatorname{Dmn}(\cdot, A)=A^{2}$. Also suppose $\lambda=\langle a, b, c, d\rangle \in A^{4}$ and $a \neq b$ and $c \equiv d\left(\Theta_{H}(a, b)\right)$. Take $p, q$, $r \notin A$ and set $\boldsymbol{A}^{\prime}=\langle A \cup\{p, q, r\}, \cdot, 0\rangle$ where $x \cdot y$ is defined (and equal $x \cdot y$ in $A)$ iff $x, y \in A$. For $\Theta \in H$ with $\rho(a, b, c, d) \supset \Theta$, set $\Theta^{\prime}=\Theta U$ $\{\langle p, p\rangle,\langle q, q\rangle,\langle r, r\rangle\}$. For $\Theta \in H$ with $\rho(a, b, c, d) \leqq \Theta$, set $\Theta^{\prime}=\Theta U$ $(0 / \Theta \cup\{p, q, r\})^{2}$. (Note that by $(\mathrm{E})$ of $(\#) \Theta^{\prime}$ is defined for each $\Theta \in$ $H$.) $\quad H^{\prime}=\left\{\Theta^{\prime}: \Theta \in H\right\}$. Set $\rho^{\prime}(p)=\rho^{\prime}(q)=\rho^{\prime}(r)=(\rho(a, b, c, d))^{\prime}$ and $\rho^{\prime}(x)=(\rho(x))^{\prime}$ if $x \in A$. Let $\Phi$ be the smallest equivalence relation on $\boldsymbol{A}^{\prime}[\cdot]$ which includes $\langle c, a p\rangle$ and $\langle b p, b q\rangle$ and $\langle a q, a r\rangle$ and $\langle b r, d\rangle$. $\Phi$ is a congruence relation of $A^{\prime}[\cdot]$ because $\left.\Phi\right|_{A^{\prime}}$ is the equality relation and $\operatorname{Dmn}\left(\cdot, \boldsymbol{A}^{\prime}[\cdot]\right)=\left(A^{\prime}\right)^{2}$. Set $\boldsymbol{A}_{\lambda}=\left(\boldsymbol{A}^{\prime}[\cdot]\right) / \Phi=\left\langle A_{\lambda}, \cdot, 0\right\rangle$. Since $\left.\Phi\right|_{A^{\prime}}=\Delta, A_{\lambda}$ is an extension of $A^{\prime}$. So we assume $A^{\prime} \cong A_{\lambda}$. For each $\Theta \in H$, let $\Theta_{\lambda}$ be the smallest congruence relation of $\boldsymbol{A}_{\lambda}$ containing $\Theta^{\prime}$. We let $H_{\lambda}=\left\{\Theta_{\lambda}: \Theta \in H\right\}$. For $x \in A^{\prime}$ we set $\rho_{\lambda}(x)=$ $\left(\left.\left(\rho^{\prime}(x)\right)\right|_{A}\right)_{\lambda}$, and for $x \in A_{\lambda}-A^{\prime}$ we set $\rho_{\lambda}(x)=\left(\Lambda\left\{\rho(c): 0 \equiv x\left((\rho(c))_{\lambda}\right)\right\}\right)_{\lambda}$.

LEMMA 8. Under the above hypotheses the following hold:

(i) $\left\langle\boldsymbol{A}^{\prime}, H^{\prime}, \rho^{\prime}\right\rangle$ is an extension of $\langle\boldsymbol{A}, H, \rho\rangle$;

(ii) $\left\langle\boldsymbol{A}_{\lambda}, H_{\lambda}, \rho_{\lambda}\right\rangle$ is an extension of $\langle\boldsymbol{A}, H, \rho\rangle$;

(iii) in $A_{\lambda}$ we have $c \equiv d(\theta(a, b))$.

The last part of the above lemma means that if $\Psi \in \operatorname{Con}\left(A_{\lambda}\right)$ and $a \equiv b(\Psi)$, then also $c \equiv d(\Psi)$.

We will prove these lemmas later.

THEOREM 2. If $L$ is a pinched lattice, then there is a pointed groupoid $\boldsymbol{A}$ satisfying the following:

(i) $\operatorname{Con}(\boldsymbol{A})$ is isomorphic to $L$; 
(ii) if $c \equiv d(\Theta(a, b))$, then there are $p, q, r \in A$ so that $c=a p$ and $b p=b q$ and $a q=a r$ and $b r=d$;

(iii) all joins in Con $\boldsymbol{A}$ are type-3.

We shall prove this theorem assuming Lemmas 4-8. We can slightly reduce our total notational complexity by first proving another lemma.

Suppose $\boldsymbol{A}$ is a pointed groupoid and $\langle\boldsymbol{A}, H, \rho\rangle$ satisfies (\#). Index $\left\{\lambda: \lambda=\langle a, b, c, d\rangle \in A^{4}, a \neq b, c \equiv d\left(\Theta_{H}(a, b)\right)\right\}$ by its cardinal number $\kappa$. Set $\langle\boldsymbol{A}, H, \rho\rangle=\left\langle\boldsymbol{A}_{0}, H_{0}, \rho_{0}\right\rangle$. Suppose $\alpha \leqq \kappa$ and $\left\langle\boldsymbol{A}_{\beta}, H_{\beta}, \rho_{\beta}\right\rangle$ has been defined for all $\beta<\alpha$ and for $\gamma<\beta<\alpha\left\langle\boldsymbol{A}_{\beta}, H_{\beta}, \rho_{\beta}\right\rangle$ is an extension of $\left\langle\boldsymbol{A}_{r}, H_{r}, \rho_{r}\right\rangle$ and $\boldsymbol{A}_{\beta}$ is a pointed groupoid for all $\beta<\alpha$. If $\alpha=\beta+1$, set $\left.\left\langle\boldsymbol{A}_{\alpha}, H_{\alpha}, \rho_{\alpha}\right\rangle=\left\langle\boldsymbol{F r}\left(\left(\boldsymbol{A}_{\beta}\right)_{\lambda_{\beta}}\right), \boldsymbol{F r}\left(\left(H_{\beta}\right)_{\lambda_{\beta}}\right)\right), \boldsymbol{F r}\left(\left(\rho_{\beta}\right)_{\lambda_{\beta}}\right)\right\rangle$. If $\alpha$ is a limit ordinal, then we let $\left\langle A_{\alpha}, H_{\alpha}, \rho_{\alpha}\right\rangle$ be given by Lemma 6. Set $\left\langle\boldsymbol{A}^{\prime \prime}, H^{\prime \prime}, \rho^{\prime \prime}\right\rangle=\left\langle\boldsymbol{A}_{\kappa}, H_{\kappa}, \rho_{\kappa}\right\rangle$.

LEMMA 9. Under the above hypotheses the following hold:

(i) $\boldsymbol{A}^{\prime \prime}$ is a pointed groupoid;

(ii) $\left\langle\boldsymbol{A}^{\prime \prime}, H^{\prime \prime}, \rho^{\prime \prime}\right\rangle$ is an extension of $\langle\boldsymbol{A}, H, \rho\rangle$;

(iii) if $a, b \in A$ and $c \equiv d\left(\Theta_{H}(a, b)\right)$; then there are $p, q, r \in A^{\prime \prime}$ so that $c=a p$ and $b p=b q$ and $a q=a r$ and $b r=d$.

(iv) if $a, b \in A$, then in $\boldsymbol{A}^{\prime \prime}$ we have $\Theta(a, b) \supseteqq \Theta_{H}(a, b)$.

REMARK. It is a general fact that if $\langle\boldsymbol{C}, K, \boldsymbol{\sigma}\rangle$ is an extension of some $\langle\boldsymbol{B}, H, \rho\rangle$, then the mapping which sends $\Theta \rightarrow[\Theta]_{K}$ is an isomorphism from $\langle H ; \cong\rangle$ onto $\langle K$; $\subseteq$. This can be proved by noting that (iii) of the definition implies $\Theta \cong \Phi$ iff $[\Theta]_{K} \subseteq[\Phi]_{K}$ (and so the mapping is an order isomorphism) and (iv) of definition implies this mapping is onto. A further consequence is that if $a, b, c, d \in B$ and $c \equiv d\left(\Theta_{H}(a, b)\right)$, then also $c \equiv d\left(\Theta_{K}(a, b)\right)$.

Proof. Using the last sentence in the remark and Lemmas 6, 7 , and 8 and transfinite induction, one can easily show for each $\alpha \leqq$ $\kappa$ that $\left\langle\boldsymbol{A}_{\alpha}, H_{\alpha}, \rho_{\alpha}\right\rangle$ exists and is an extension of each $\left\langle\boldsymbol{A}_{\beta}, H_{\beta}, \rho_{\beta}\right\rangle$ with $\beta \leqq \alpha$ and that $\boldsymbol{A}_{\alpha}$ is a pointed groupoid. Thus (i) and (ii) hold.

Let $a, b \in A$, and let $c \equiv d\left(\Theta_{H}(a, b)\right)$. Suppose $a \neq b$. Then since $\langle a, b, c, d\rangle$ is some $\lambda_{\beta}$ we have the required $p, q, r$. If $\boldsymbol{A}$ is the one element algebra, we may (and must) take $p=q=r=0$. Suppose $|A| \geqq 2$ and $a=b$. Then, since $\Delta \in H, c=d$. Choose any $b^{\prime} \neq a$. Then $\left\langle a, b^{\prime}, c, d\right\rangle$ is some $\lambda_{\beta}$. So in $\boldsymbol{A}^{\prime \prime}$ there is a $p$ with $a p=c=d$. So in this case we let $q=r=p$. Thus (iii) holds. (iv) follows easily from (iii).

Proof of Theorem 2. Let $L$ be a pinched lattice. Set $\left\langle A_{0}, H_{0}, \rho_{0}\right\rangle=$ 
$\langle\boldsymbol{F r}(B), \boldsymbol{F r}(H), \boldsymbol{F r}(\rho)\rangle$ where $\langle\boldsymbol{B}, H, \rho\rangle$ is given by Lemma 4 . Set $\left\langle\boldsymbol{A}_{n+1}, H_{n+1}, \rho_{n+1}\right\rangle=\left\langle\boldsymbol{A}_{n}^{\prime \prime}, H_{n}^{\prime \prime}, \rho_{n+1}^{\prime \prime}\right\rangle$ using the construction for Lemma 9. Consider $\left\langle\boldsymbol{A}_{\omega}, H_{\omega}, \rho_{\omega}\right\rangle$ as given by Lemma 6 . We set $\boldsymbol{A}=\boldsymbol{A}_{\omega}$. Since $\boldsymbol{A}$ is a direct limit of pointed groupoids, $\boldsymbol{A}$ is also one. Lemma 4, Lemma 7, Lemma 9, Lemma 6, induction, the transitivity of the extension relation, and the remark after Lemma 9 all imply that $\left\langle H_{\omega} ; \cong\right\rangle \cong\langle H ; \subseteq\rangle \cong C(L)$, the semilattice of compact elements of $L$.

We claim that Con $\boldsymbol{A}$ is isomorphic to $L$; i.e., we claim (i) holds. It suffices to show that the semilattice of finitely generated congruences is isomorphic to $C(L)$; i.e., it suffices to show that $H_{\omega}$ is the set of finitely generated congruences of $\boldsymbol{A}$. By the definition of basis and $(\mathrm{K})$ of (\#), it suffices to show that in $\boldsymbol{A}$ we have $\Theta(a, b)=$ $\Theta_{H_{\omega}}(a, b)$ for each $a, b \in A$.

If $a, b \in A_{n}$, we let $\Theta_{n}(a, b)$ denote the smallest congruence relation of $\boldsymbol{A}_{n}$ containing $\langle a, b\rangle$. By (iv) of Lemma 9 and induction, by Lemma 9 and Lemma 6 and the remark after Lemma 9, and from general principles we have, for each $a, b \in A$, that $\Theta_{H_{\omega}}(a, b) \supseteqq \Theta(a, b) \supseteqq$ $\left.\mathrm{U} \Theta_{n+1}(a, b): a, b \in A_{n}\right) \supseteqq \bigcup \bigcup\left(\Theta_{H_{n}}(a, b): a, b \in A_{n}\right)=\Theta_{H_{\omega}}(a, b)$. But this is what we were required to prove. Also, (ii) of Theorem 2 now follows easily from (iii) of Lemma 9. We defer the proof of (iii) till after the proof of Lemma 4.

Proof of Lemma 4. We suppose $L$ is a pinched lattice. Set $B=C(L)$, and let 0 be the zero of $C(L)$. Set $0 \cdot 0=0$ and $\operatorname{Dmn}(\cdot)=$ $\{\langle 0,0\rangle\}$ and $\boldsymbol{B}=\langle B, \cdot, 0\rangle$. For $b \in B$ define $\Theta_{b}$ by $x \equiv y\left(\Theta_{b}\right)$ iff $x=y$ or $x \vee y \leqq b$. We set $H=\left\{\Theta_{b}: b \in B\right\}$. Let $I$ be the set given by Proposition 1. Define $\sigma: B \rightarrow I$ by $\sigma(b)=\Lambda\{i \in I: b \leqq i\}$. Then set $\rho(b)=\Theta_{\sigma(b)}$. It is not too hard to show that $\langle\boldsymbol{B}, H, \rho\rangle$ has the required properties. Details are left to the reader.

Proof of (iii) of Theorem 2. Let $c, d \in A$ and $\Theta, \Phi \in \operatorname{Con}(A)$. Suppose $c \equiv d(\Theta \vee \Phi)$ and $c \neq \equiv d(\Theta)$ and $c \neq \equiv d(\Phi)$. Since $\Theta(c, d)$ is compact in $\operatorname{Con}(\boldsymbol{A})$, we can find compact $\Theta_{0}, \Phi_{0}$ such that $c \equiv d\left(\Theta_{0} \vee \Phi_{0}\right)$ and $\Theta_{0} \subseteq$ $\Theta$ and $\Phi_{0} \subseteq \Phi$. For the $B$ and $H$ of the proof of Lemma 4 we have $B \subseteq A$ and $\left.\Theta_{0}\right|_{B} \in H$ and $\left.\Phi_{0}\right|_{B} \in H$. So there exist $a, b \in B$ with $\left.\Theta_{0}\right|_{B}=$ $\Theta_{H}(a, 0)$ and $\left.\Phi_{0}\right|_{B}=\Theta_{H}(b, 0)$ and $\Theta_{H}(a, b)=\left.\left.\Theta_{0}\right|_{B} \vee \Phi_{0}\right|_{B}$. Hence $\Theta_{0}=\Theta(a, 0)$ and $\Phi_{0}=\Theta(b, 0)$ and $\Theta_{0} \vee \Phi_{0}=\Theta(a, b)$. Thus we have $c \equiv d(\Theta(a, b))$. By (ii) there exist $p, q, r$ such that $c=a p \equiv 0 p\left(\Theta_{0}\right)$ and $0 p \equiv b p=$ $b q \equiv 0 q\left(\Phi_{0}\right)$ and $0 q \equiv a q=a r \equiv 0 r\left(\Theta_{0}\right)$ and $0 r \equiv b r=d\left(\Phi_{0}\right)$. That is, $\langle c, d\rangle \in \Theta \circ \Phi \circ \Theta \circ \Phi$.

Proof of Lemma 5. By Lemma $2.5 \Delta_{A}[\cdot]=\Delta_{A[\cdot]}$. So (A) and (B) of (\#) hold for $A[\cdot]$ and $H[\cdot]$. 
It follows from Lemma 2.5 that $\left.\Theta[\cdot]\right|_{A}=\Theta$ for any $\Theta \in \operatorname{Con}(A)$ and that $H[\cdot] \subseteq \operatorname{Con}(A[\cdot])$. It follows that $\langle H[\cdot] ; \subseteq\rangle$ is isomorphic to the semilattice $\langle H ; \cong\rangle$.

Lemma 2.5, (\#) and Lemma 3 imply that $\Theta_{H[\cdot]}(e, f)$ exists for each $e, f \in A[\cdot]$ and that the following hold for $\Theta_{H[\cdot] \cdot]}(e, f)$ :

(i) if $e, f \in A$, then $\Theta_{H[\cdot]}(e, f)=\left(\Theta_{H}(e, f)\right)[\cdot]$;

(ii) if $e \in A$ and $f=v y \notin A$ and $r \operatorname{CLS} v$ (in $D, \bmod H$ ) and $t$ CLS $y($ in $D, \bmod H)$, then $\Theta_{H[\cdot]}(e, f)=\left(\Theta_{H}(e, r t) \vee \Theta_{H}(r, v) \vee \Theta_{H}(t, y)\right)[\cdot]$;

(iii) if $e=u x \notin A$ and $f=v y \notin A$, then $\Theta_{H[\cdot]}(e, f)$ is the smaller of $\Phi(u x, v y)[\cdot]$ and $\Psi(u x, v y)[\cdot]$. (These notations are from before Lemma 3.)

Lemma 2.2 applied to $H$, that $H[\cdot]=\{\Theta[\cdot]: \Theta \in H\}$, and the above imply that $H[\cdot]$ satisfies (i)-(iii) of Lemma 2.2. We conclude that $H[\cdot]$ is a basis. So (C) of (\#) holds.

That (D) of (\#) holds for $\rho[\cdot]$ and $H[\cdot]$ follows easily from the isomorphism between $\langle H ; \subseteq\rangle$ and $\langle H[\cdot] ; \subseteq\rangle$ and from the definition of $\rho[\cdot]$ and that it holds for $\rho$ and $H$. Similarly for (E) and (F) of (\#).

$A \times A=\operatorname{Dmn}(\cdot, A[\cdot])$. So (G) of (\#) holds.

If $a \in A$, then certainly $a \operatorname{CLS} a$ (in $A, \bmod H[\cdot])$. If $a=v y \notin A$, then $r t$ CLS $a$ (in $A, \bmod H[\cdot])$ for any $r, t$ satisfying $r$ CLS $v$ (in $D$, $\bmod H)$ and $t$ CLS $y($ in $D, \bmod H)$. So $(\mathrm{H})$ of (\#) holds.

Consider (I). Let $e, f \in A[\cdot]$. If $e, f \in A$, then $\Theta_{H[\cdot]}(e, f) \supseteqq$ $\Theta_{H[\cdot]}(e, f)$ will suffice. If $e \in A$ and $f \notin A$, we established above there is a $g$ with $g$ CLS $f$ (in $A, \bmod H[\cdot])$. It follows that $\Theta_{H[\cdot]}(e, f) \supseteqq$ $\Theta_{H[\cdot]}(f, g)$. Hence $\Theta_{H[\cdot]}(e, f) \supseteqq \Theta_{H[\cdot]]}(e, g)$. Let $e=u x \notin A$ and $f=v y \notin$ $A$. Using (I) of (\#) for $\boldsymbol{A}$ and $\boldsymbol{H}$, choose $q, r, s, t$ satisfying $q$ CLS $u$ (in $D, \bmod H$ ), etc., and $\Theta_{H}(u, v) \supseteqq \Theta_{H}(q, r)$ and $\Theta_{H}(x, y) \supseteqq \Theta_{H}(s, t)$. So $\Phi(u x, v y) \supseteqq \Theta_{H}(q, r) \vee \Theta_{H}(s, t) \supseteqq \Theta_{H}(q s, r t)$. Thus we have $\Theta_{H[\cdot]}(e, f) \supseteqq$ $\Theta_{H}(q s, r t)$. In the preceding paragraph we established that $q s$ CLS $e$ (in $A, \bmod H[\cdot])$ and $r t \operatorname{CLS} f(\operatorname{in} A, \bmod H[\cdot])$. So (I) of (\#) holds.

Given Lemma 3 and descriptions of the $\Theta_{H[\cdot]}(e, f)$ and that (J) holds for $\boldsymbol{A}$ and $H$, it is easy to show that $(J)$ holds for $A[\cdot]$ and $H[\cdot]$. There are three cases: (i) $u x, v y \in A$; (ii) $u x \in A$ and $v y \notin A$; (iii) $u x \notin A$ and $v y \notin A$. (i) and (iii) are left to the reader. Suppose $u x \in A$ and $v y \notin A$. Choose $r, t$ satisfying $r \operatorname{CLS} v($ in $D, \bmod H)$ and $t$ CLS $y($ in $D, \bmod H)$. Then $\Theta_{H[\cdot \cdot]}(u x, v y)=\left(\Theta_{H}(u x, r t) \vee \Phi(r t, v y)\right)[\cdot]$. The case $\Theta_{H}(u x, r t)=\Phi(u x, r t)$ is easy. Suppose $\Theta_{H}(u x, r t) \neq \Phi(u x, r t)$. If $\Phi(r t, v y) \supseteqq \rho(u, x, r, t)$, then $\Theta_{H[\cdot]}(u x, v y) \supseteqq \Phi(r t, v y)[\cdot] \supseteqq \Phi(u x, v y)[\cdot] \supseteqq$ $\Theta_{H[\cdot]}(u x, v y)$. So we may suppose by (E) of (\#) that $\Phi(r t, v y) \leqq$ $\rho(u, x, r, t)$. Now $(J)$ for $A$ and $H$ implies $\Theta_{H}(x, y)=\Theta_{H}(x, t)=$ $\rho(u, x, r, t)=\rho(u, x, v, y)$.

Since $H$ is a basis, each member of $H$ is the closure of some finite subset of $A \times A$. So (K) of (\#) for $A[\cdot]$ and $H[\cdot]$ follows 
easily from (K) of (\#) for $A$ and $H$ and from $\Theta \rightarrow \Theta[\cdot]$ being an order isomorphism.

The details for showing that $\langle A[\cdot], H[\cdot], \rho[\cdot]\rangle$ is an extension of $\langle\boldsymbol{A}, H, \rho\rangle$ either are easy or appear above.

Proof of Lemma 6. Suppose $x, y \in A_{\alpha}$. Then there is a $\beta<\alpha$ with $x, y \in A_{\beta}$. (ii) of the definition of extension implies $x \cdot y$ is defined in $\boldsymbol{A}_{\beta+1}$. So $\boldsymbol{A}_{\alpha}$ is a pointed groupoid. Let $\gamma$ be the least ordinal with $x, y \in A_{\gamma}$. We note that $\Theta_{H_{\alpha}}(x, y)=\left(\left.\left(\Theta_{H_{\gamma}}(x, y)\right)\right|_{A_{0}}\right)_{\alpha}=$ $\mathbf{U}\left(\Theta_{H_{\beta}}(x, y): \gamma \leqq \beta<\alpha\right)$. Obviously $\Delta_{A_{\alpha}}=\left(\Delta_{A_{0}}\right)_{\alpha}$ and $H_{\alpha} \leqq \operatorname{Con} \boldsymbol{A}_{\alpha}$. $\left\langle H_{\alpha} ; \cong\right\rangle$ is isomorphic to the semilattice $\left\langle H_{0} ; \cong\right\rangle$. Lemma 2.2 applied to $H_{0}$, the definition of extension, that $H_{\alpha}=\left\{\Theta_{\alpha}: \Theta \in H_{0}\right\}$, and the above show that (i)-(iii) of Lemma 2.2 hold for $H_{\alpha}$. We conclude that $H_{\alpha}$ is a basis. Since $\rho_{\alpha}: A_{\alpha} \rightarrow H_{\alpha}$, we have that (A), (B), and (C) of (\#) hold.

Let $\sigma: A_{0} \rightarrow H_{\alpha}$ be defined by $\sigma(a)=\left(\rho_{0}(a)\right)_{\alpha}$. Clearly $\operatorname{Rng}(\sigma)=$ $\operatorname{Rng}\left(\rho_{\alpha}\right)$ because of (v) and (vi) of the definition of "extension." Now $(\mathrm{D}),(\mathrm{E})$ and $(\mathrm{F})$ of $(\#)$ follow easily.

$A_{\alpha} \times A_{\alpha}=\operatorname{Dmn}\left(\cdot, \boldsymbol{A}_{\alpha}\right)$, and $(\mathrm{G})$ of ( $($ ) holds. Since $x$ CLS $x$ (in $A_{\alpha}, \operatorname{Mod} H_{\alpha}$ ) holds for every $x \in A_{\alpha},(\mathrm{H})$ and (I) of (\#) hold.

Suppose $\gamma, \delta, \varepsilon$ are the least ordinals satisfying $u x, v y \in A_{\gamma}$ and $u, v \in A_{\delta}$ and $x, y \in A_{\varepsilon}$. Note that $\delta, \varepsilon \leqq \gamma$. Now

$$
\begin{aligned}
\Theta_{H_{\alpha}}(u x, v y) & =\left(\left.\left(\Theta_{H_{\gamma}}(u x, v y)\right)\right|_{A_{0}}\right)_{\alpha}=\left(\left.\left(\Theta_{H_{\gamma}}(u, v) \vee \Theta_{H_{\gamma}}(x, y)\right)\right|_{A_{0}}\right)_{\alpha} \\
& =\left(\left.\left.\left(\Theta_{H_{\gamma}}(u, v)\right)\right|_{A_{0}} \vee \Theta_{H_{\gamma}}(x, y)\right|_{A_{0}}\right)_{\alpha} \\
& =\left(\left.\left.\left(\Theta_{H_{\hat{o}}}(u, v)\right)\right|_{A_{0}} \wedge\left(\Theta_{H_{\varepsilon}}(x, y)\right)\right|_{A_{0}}\right)_{\alpha} \\
& =\left(\left.\left(\Theta_{H_{\hat{o}}}(u, v)\right)\right|_{A_{0}}\right)_{\alpha} \vee\left(\left.\left(\Theta_{H_{\varepsilon}}(x, y)\right)\right|_{A_{0}}\right)_{\alpha} \\
& =\Theta_{H_{\alpha}}(u, v) \vee \Theta_{H_{\alpha}}(x, y)
\end{aligned}
$$

or $\Theta_{H_{\alpha}}(x, y)=\left(\left.\left(\Theta_{H_{\varepsilon}}(x, y)\right)\right|_{A_{0}}\right)_{\alpha}=\left(\left.\left(\Theta_{H_{\gamma}}(x, y)\right)\right|_{A_{0}}\right)_{\alpha}=\left(\left.\left(\rho_{\gamma}(u, v, x, y)\right)\right|_{A_{0}}\right)_{\alpha}=$ $\rho_{\alpha}(u, v, x, y)$. So $(J)$ of (\#) holds.

The proof that $(K)$ of $(\#)$ holds is similar to the proof of the corresponding part of Lemma 5 .

Note that if $\Phi \in H_{\beta}$, then $\Phi=\left[\left.\Phi\right|_{A_{0}}\right]_{H_{3}}$ because $\left\langle\boldsymbol{A}_{\beta}, H_{\beta}, \rho_{\beta}\right\rangle$ is an extension $\left\langle\boldsymbol{A}_{0}, H_{0}, \rho_{0}\right\rangle$.

It is now easy to check that $\left\langle\boldsymbol{A}_{\alpha}, H_{\alpha}, \rho_{\alpha}\right\rangle$ is an extension of $\left\langle\boldsymbol{A}_{\beta}, H_{\beta}, \rho_{\beta}\right\rangle$ for each $\beta \leqq \alpha$.

Proof of Lemma 7. This is a corollary of Lemmas 5 and 6.

Proof of Lemma 8. We assume (i), the proof of which is quite straightforward.

Set $X=\{x \in A: \rho(x) \leqq \rho(a, b, c, d)\}$ and $Y=A-X$. Observe that 
Claim 1. If $x, x_{0}, x_{1} \in X$ and $y \in Y$, then:

(i ) $\Theta_{H}\left(x_{0}, x_{1}\right) \subseteq \rho(a, b, c, d)$;

(ii) $\Theta_{H}(x, y) \supsetneq \rho(a, b, c, d)$;

(iii) $\left.\rho(a, b, c, d)\right|_{X}=X \times X$.

It then follows easily from Lemma 2.5 and the definition of the map $\Theta \rightarrow \Theta^{\prime}$ that

Claim 2. If, in $A^{\prime}[\cdot], x$ belongs to one of the sets $A^{\prime}$, $\{p, q, r\} \cdot\{p, q, r\}, X p, p X, X q, q X, r X, X r, Y p, p Y, Y q, q Y, Y r, r Y$ and $y$ belongs to a different one, then $\Theta_{H^{\prime}[\cdot]}(x, y) \supseteqq \rho^{\prime}[\cdot](a, b, c, d)$.

Set $T$ (for trash) $=A^{\prime}[\cdot]-\left(A^{\prime} \cup X p \cup X q \cup X r\right)$. We clearly have

Claim 3. If $x$ and $y$ are in different blocks of the partition $\left\{A^{\prime}, T, X p, X q, X r\right\}$, then $\Theta_{H^{\prime}[\cdot]}(x, y) \supseteqq \rho^{\prime}[\cdot](a, b, c, d)$.

$A^{\prime}[\cdot]$ is pictured in Figure 2 and $A_{\lambda}$ is pictured in Figure 3.

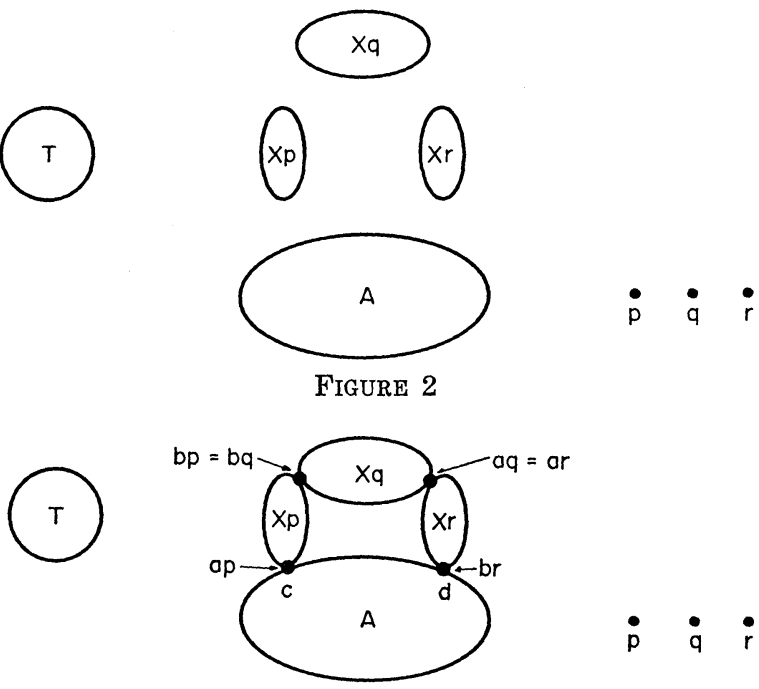

FIGURE 3

Recall the relation $\Phi$ used in the definition of $A_{\lambda}$. Observe that $\Phi \subseteq(\rho(a, b, c, d))^{\prime}[\cdot]$.

In what follows we let + denote equivalence relation join. ${ }_{+}+$ need not coincide with join in $\operatorname{Con}\left(\boldsymbol{A}^{\prime}[\cdot]\right)$.) Thus we have

Claim 4. If $\Theta \in H$ and $\Theta \supseteqq \rho(a, b, c, d)$, then $\left(\Theta^{\prime}[\cdot]+\Phi\right)=\Theta^{\prime}[\cdot]$.

In order to establish that $H_{\lambda}$ is a basis and that each $\left.\Theta_{\lambda}\right|_{A}=\Theta$, we need a fairly detailed description of $\Theta_{\lambda}$. We will show for each $\Theta \in H$ that $\left(\Theta^{\prime}[\cdot]+\Phi\right) \in \operatorname{Con}\left(A^{\prime}[\cdot]\right)$. From this it follows on general 
principles that $\Theta_{2}=\left(\Theta^{\prime}[\cdot]+\Phi\right) / \Phi$. So we will profit from an examination of $\Theta^{\prime}[\cdot]+\Phi$.

Claim 5. The following hold for $x, y \in A^{\prime}[\cdot]$ and $\Theta \in H$ :

(i) if $x$ and $y$ both belong to one of the sets $A^{\prime} \cup T, X p \cup T$, $X q \cup T, X r \cup T$, then $x \equiv y\left(\Theta^{\prime}[\cdot]+\Phi\right)$ iff $x \equiv y\left(\Theta^{\prime}[\cdot]\right)$.

(ii) if $x \in A^{\prime}$ and $y \in X p$, then $x \equiv y\left(\Theta^{\prime}[\cdot]+\Phi\right)$ iff $x \equiv c\left(\Theta^{\prime}[\cdot]\right)$ and $a p \equiv y\left(\Theta^{\prime}[\cdot]\right)$;

(iii) if $x \in A^{\prime}$ and $y \in X r$, then $x \equiv y\left(\Theta^{\prime}[\cdot]+\Phi\right)$ iff $x \equiv d\left(\Theta^{\prime}[\cdot]\right)$ and $b r \equiv y\left(\Theta^{\prime}[\cdot]\right)$;

(iv) if $x \in A^{\prime}$ and $y \in X q$, then $x \equiv y\left(\Theta^{\prime}[\cdot]+\Phi\right)$ iff $x \equiv c\left(\Theta^{\prime}[\cdot]\right)$ and $a=b\left(\Theta^{\prime}[\cdot]\right.$ and $b q \equiv y\left(\Theta^{\prime}[\cdot]\right)$;

$(\mathrm{v})$ if $x \in X p$ and $y \in X q$, then $x \equiv y\left(\Theta^{\prime}[\cdot]+\Phi\right)$ iff $x \equiv b p\left(\Theta^{\prime}[\cdot]\right)$ and $b q \equiv y\left(\Theta^{\prime}[\cdot]\right)$;

(vi) if $x \in X q$ and $y \in X r$, then $x \equiv y\left(\Theta^{\prime}[\cdot]+\Phi\right)$ iff $x \equiv a q\left(\Theta^{\prime}[\cdot]\right)$ and $a r \equiv y\left(\Theta^{\prime}[\cdot]\right)$;

(vii) if $x \in X p$ and $y \in X r$, then $x \equiv y\left(\Theta^{\prime}[\cdot]+\Phi\right)$ iff $x \equiv a p\left(\Theta^{\prime}[\cdot]\right)$ and $c \equiv d\left(\Theta^{\prime}[\cdot]\right)$ and $b r \equiv y\left(\Theta^{\prime}[\cdot]\right)$.

The proof of the claim is quite routine, and so we leave most of the proof to the reader. But we prove part (i) as an example.

It is obvious that if $x \equiv y\left(\Theta^{\prime}[\cdot]\right)$, then $x \equiv y\left(\Theta^{\prime}[\cdot]+\Phi\right)$. So we suppose $x \equiv y\left(\Theta^{\prime}[\cdot]+\Phi\right)$ and $x$ and $y$ both belong to one of the sets $A^{\prime} \cup T, X p \cup T, X q \cup T, X r \cup T$. If $\Theta \supseteqq \rho(a, b, c, d)$, then by Claim 4 $x \equiv y\left(\Theta^{\prime}[\cdot]\right)$. So we suppose $\Theta \varsubsetneqq \rho(a, b, c, d)$.

Let $x_{0} \in T, x_{1} \in A^{\prime}, x_{2} \in X p, x_{3} \in X q, x_{4} \in X r$. By Claim 3 we have

$$
x_{i} \not \equiv x_{j}(\Theta[\cdot]) \text { if } i \neq j .
$$

Since $x \equiv y\left(\Theta^{\prime}[\cdot]+\Phi\right)$, there is a sequence $x=s_{0}, \cdots, s_{n}=y$ with $s_{i} \equiv s_{i+1}\left(\Theta^{\prime}[\cdot]\right)$ or $s_{i} \equiv s_{i+1}(\Phi)$, for $i=0, \cdots, n-1$. Suppose $s_{0}, \cdots, s_{n}$ is a sequence of shortest length having these properties. Then $s_{i} \neq s_{j}$ if $i \neq j$. If $i \leqq n-2$, then $s_{i} \equiv s_{i+1}\left(\Theta^{\prime}[\cdot]\right)$ if and only if $s_{i} \not \equiv s_{i+1}(\Phi)$ iff $s_{i+1} \equiv s_{i+2}(\Phi)$.

Let us suppose $n \geqq 2$.

Let us also suppose $x, y \in A^{\prime} \cup T$.

Now $x=s_{0} \equiv s_{1}(\Phi)$ or $s_{1} \equiv s_{2}(\Phi)$. So we take $k$ to be the least integer such that $s_{k} \equiv s_{k+1}(\Phi)$. Note that $x \equiv s_{k}\left(\Theta^{\prime}[\cdot]\right)$ and $s_{k} \neq s_{k+1}$. From the fact that $\theta \varsubsetneqq \rho(a, b, c, d)$ and $\left(^{*}\right)$ and the definition of $\Phi$, we may conclude that $x \in A^{\prime}$ and $s_{k} \in\{c, d\}$. Suppose $s_{k}=c$. The definition of $\Phi$ implies $s_{k+1}=a p$. Since $y \notin X p, y \neq s_{k+1}$ and $k+1 \neq$ $n$. So there is an $s_{k+2}$ and $s_{k+1} \equiv s_{k+2}\left(\Theta^{\prime}[\cdot]\right)$. Since $\Theta \varsubsetneqq \rho(a, b, c, d)$, Claim 3 yields that $s_{k+2} \in X p$ and $s_{k+2} \neq y$ and $k+2 \neq n$. So there is an $s_{k+3}$ with $s_{k+2} \equiv s_{k+3}(\Phi)$ and $s_{k+1} \neq s_{k+3} \neq s_{k+2}$. Now since $s_{k+2} \in X p$ and $s_{k+2} \neq s_{k+1}=a p$, the definition of $\Phi$ yields $s_{k+2} \in(\{a p, b p\})-\{a p\}=$ 
$\{b p\}$; i.e., $s_{k+2}=b p$. Continuing in this fashion we find that $s_{k+3}=b q$ and $s_{k+4}=a q$ and $s_{k+5}=a r$ and $s_{k+8}=b r$ and $s_{k+7}=d$ (and $k+7 \leqq n$ ). Keep in mind that $a p=s_{k+1} \equiv s_{k+2}=b p\left(\Theta^{\prime}[\cdot]\right)$. Now (iii) of Lemma 2.5 implies $a \equiv b\left(\Theta^{\prime}\right)$ or else there exist $e, f, u, v \in A$ (note $A^{2}=$ $\left.\operatorname{Dom}\left(\cdot, \boldsymbol{A}^{\prime}\right)\right)$ with $a \equiv e\left(\Theta^{\prime}\right)$ and $b \equiv f\left(\Theta^{\prime}\right)$ and $e \cdot u \equiv f \cdot v\left(\Theta^{\prime}\right)$ and $u \equiv$ $p \equiv v\left(\Theta^{\prime}\right)$. The latter fact implies that if $a \neq \equiv\left(\Theta^{\prime}\right)$, then $\rho(a, b, c, d) \subseteq$ $\Theta$. Hence $a \equiv b\left(\Theta^{\prime}\right)$. By construction $a \equiv b(\Theta) . \quad \Theta \in H$ and the hypotheses imply $c \equiv d(\Theta)$. So $c \equiv d\left(\Theta^{\prime}[\cdot]\right)$. Thus $x=s_{0} \equiv c \equiv d=$ $s_{k+7}\left(\Theta^{\prime}[\cdot]\right)$. As a result we see that $x=s_{0}, d=s_{k+7}, s_{k+8}, \cdots, s_{n}=y$ is a sequence in which consecutive members are equivalent under $\Theta^{\prime}[\cdot]$ or $\Phi$. Yet it is shorter than the shortest such sequence connecting $x$ and $y$. The case with $s_{k}=d$ is similar.

Let $x, y \in X p \cup T$. Suppose $x \in T$. As above we find that $x \equiv$ $s_{k}\left(\Theta^{\prime}[\cdot]\right)$, where $s_{k} \in\{c, a p, b p, b q, a q, a r, b r, d\}$. But Claim 3 implies $x \neq s_{k}\left(\Theta^{\prime}[\cdot]\right)$. So $x \notin T$. Similarly $y \notin T$. So $x, y \in X p$. Now one can proceed as in the above case and derive a contradiction.

Similar contradictions can be derived for the cases $(x, y \in X q \cup T)$ and $x, y \in(X r \cup T)$.

So $n \geqq 2$.

If $n=0$ or $n=1$ and $s_{0} \equiv s_{1}(\Phi)$, we find that $x=s_{0}=s_{1}=y$ $\left(x \equiv y(\Phi)\right.$ implies $x=y$ because $x, y \in A^{\prime} \cup T$ or $x, y \in X p \cup T$, etc.). In this case $x \equiv y\left(\Theta^{\prime}[\cdot]\right)$. The only remaining possibility is $n=0$ or 1 and $x_{0}=s_{0} \equiv s_{1}=y\left(\Theta^{\prime}[\cdot]\right)$.

This conclude our proof of (i). As stated above, the remainder of the claim is left to the reader. While doing the remainder, keep in mind that if $x \in X$ and $\Theta \in H$ and $\rho(a, b, c, d) \subseteq \theta$, then under $\Theta^{\prime}[\cdot]$ we have the following congruences

$$
\begin{aligned}
x \equiv 0 \equiv a & \equiv b \equiv c \equiv d \equiv 0 \equiv p \equiv q \equiv r \equiv 0 \\
& =0 \cdot 0 \equiv x \cdot p \equiv x \cdot q \equiv x \cdot r .
\end{aligned}
$$

By Claim 5.(i) we have $\left.\left(\Theta^{\prime}[\cdot]+\Phi\right)\right|_{A^{\prime}}=\Theta^{\prime}$ for any $\Theta \in H$. Then it clearly follows that $\Theta[\cdot]+\Phi \in \operatorname{Con}\left(A^{\prime}[\cdot]\right)$. From general principles we have that $\Theta_{\lambda}=\left(\Theta^{\prime}[\cdot]+\Phi\right) / \Phi$ for each $\Theta \in H$. That $H_{\lambda}$ is a basis is now easily proved using Lemma 2.2 and Lemma 2.4. Since $\left.\Phi\right|_{A}=$ $\Delta_{A}$, Claim 5.(i) implies $[\Theta]_{H_{\lambda}} \cap(A \times A)=\left(\Theta^{\prime}[\cdot]\right) \cap(A \times A)=\Theta$ for each $\Theta \in H$.

It is now clear that (A)-(G) of (\#) hold and that (i)-(vi) of the definition of extension hold.

In order to complete the proof, we need descriptions for $\Theta_{H_{\lambda}}(x, y)$ for $x$ and $y$ in various subsets of $A_{\lambda}$. Essentially, Claim 5 provides us with these descriptions.

From (i) of this lemma and Lemma 5 we know that for any $x \in A^{\prime}[\cdot]$ there is a $z$ with $z \operatorname{CLS} x$ (in $\left.A^{\prime}, \bmod H^{\prime}[\cdot]\right)$. From Claim 
5.(i) it follows, for $x \in A^{\prime} \cup T$, that $z \operatorname{CLS} x$ (in $A^{\prime}$, $\bmod H^{\prime}[\cdot]$ ) iff $z$ CLS $x$ (in $A^{\prime}, \bmod H_{\lambda}$ ). So for each $x \in A^{\prime} \cup T$ we have the required z. Claim 5 ensures the following: if $x \in(X p \cup X q)$, then $c$ CLS $x$ (in $A^{\prime}, \bmod H_{\lambda}$ ); if $x \in(X q \cup X r)$, then $d \operatorname{CLS} x$ (in $A^{\prime}, \bmod H_{\lambda}$ ). (We remind the reader that closest elements need not be unique.) So $(\mathrm{H})$ of $(\#)$ holds.

Consider (I) of ( $\#)$.

Suppose $x, y \in A^{\prime} \cup T$. By (i) of this lemma and Lemma 5 and the preceding paragraph there exist $u, v$ such that $\Theta_{H_{\lambda}}(x, y)=$ $\left(\Theta_{H^{\prime}[\cdot]}(x, y)+\Phi\right) / \Phi \supseteq\left(\Theta_{H^{\prime}[\cdot]}(u, v)+\Phi\right) / \Phi=\Theta_{H_{\lambda}}(u, v)$ and $u$ CLS $x$ (in $A^{\prime}$, $\left.\bmod H_{\lambda}\right)$ and $v$ CLS $y\left(\right.$ in $A^{\prime}, \bmod H_{\lambda}$.

Suppose $x \in T$ and $y \in X p \cup X q \cup X r$. Then

$$
\Theta_{H_{\lambda}}(x, y)=\left(\Theta_{\left.H^{\prime}[\cdot]\right]}(x, y)+\Phi\right) / \Phi \text {. }
$$

By Claim 3 we have $\Theta_{H^{\prime}[\cdot]}(x, y) \supseteqq \rho^{\prime}[\cdot](a, b, c, d)$. By Claims 1.(iii) and 4 we have $c \equiv y \equiv d\left(\Theta_{H^{\prime}[\cdot]}(x, y)\right)$. Let $v=c$ or $d$ as appropriate and choose any $u$ satisfying $u \operatorname{CLS} x$ (in $A^{\prime}, \bmod H_{\lambda}$ ). So $x \equiv v$ $\left(\Theta_{H^{\prime}[\cdot]}(x, y)\right)$. We clearly have $\Theta_{H_{\lambda}}(x, y) \supseteqq \Theta_{H_{\lambda}}(x, v) \supseteqq \Theta_{H_{\lambda}}(u, v)$ since $v \in A^{\prime}$.

Suppose $x \in A^{\prime}$ and $y \in X p \cup X q \cup X r$. Above we established that there is a $z$ satisfying $z \operatorname{CLS} y$ (in $A^{\prime}, \bmod H_{\lambda}$ ). We have then $\Theta_{H_{2}}(x, y) \supseteqq \Theta_{H_{\lambda}}(x, z)$. Note that $x$ is closest to itself.

If $x, y \in(X p \cup X q)$, then $\Theta_{H_{\lambda}}(x, y) \supseteqq \Delta=\Theta_{H_{\lambda}}(c, c)$ will suffice. Similarly for $x, y \in(X q \cup X r)$.

If $x \in X p$ and $y \in X r$ or vice versa, then Claim 5 implies $\Theta_{H_{\lambda}}(x, y) \supseteqq \Theta_{H_{\lambda}}(c, d)$. But $c$ is closest to $x$ and $d$ is closest to $y$ (or vice versa).

If $x \in A^{\prime}$, then $x$ is closest to itself. So if $x, y \in A^{\prime}, \Theta_{H_{\lambda}}(x, y) \supseteqq$ $\Theta_{H \lambda}(x, y)$ will suffice.

So (I) of (\#) holds.

Consider $(\mathrm{J})$ of (\#) and let $\{u, v, x, y\} \subseteq A^{\prime}$. There are the following nondisjoint cases:

(i) $\{u x, v y\} \cong A^{\prime} \cup T$ and $\{\langle u, x\rangle,\langle v, y\rangle\} \cap\{\langle a, p\rangle,\langle b, r\rangle\}=\varnothing$;

(ii) $x=y$;

(iii) $x \neq y$ and $x \in\{p, q, r\}$ and $u, v, y \in(X \cup\{p, q, r\})$;

(iv) $x \neq y$ and $y \in\{p, q, r\}$ and $u, v, x \in(X \cup\{p, q, r\})$;

(v) $x \neq y$ and $x \in\{p, q, r\}$ and $u \in X$ and $\{v, y\} \nsubseteq X \cup\{p, q, r\}$;

(vi) $x \neq y$ and $y \in\{p, q, r\}$ and $v \in X$ and $\{u, x\} \nsubseteq X \cup\{p, q, r\}$.

(i)-(iv) are easy to check, and (vi) follows from (v) since, in general, $\Theta_{H_{\lambda}}(w, z)=\Theta_{H_{\lambda}}(z, w)$. So we shall prove $(\mathrm{J})$ under the additional hypotheses in case $(\mathrm{v})$.

Observe that $x \equiv 0 \equiv y\left(\Theta_{H_{\lambda}}(x, y)\right)$. We may assume $\Theta_{H_{\lambda}}(x, y) \varsubsetneqq \rho_{\lambda}(u$, $v, x, y)$. Note that $\rho_{\lambda}(u) \subseteq \rho_{\lambda}(x)=\Theta_{H_{\lambda}}(x, 0) \subseteq \Theta_{H_{\lambda}}(x, y)$. Since one of $v$ and $y$ is in $Y,(\mathrm{E})$ of (\#) now implies $\rho_{\lambda}(u, v, x, y)=\rho_{\lambda}(v, y)$. Since 
$\Theta_{H_{\lambda}}(y, 0) \subseteq \Theta_{H_{\lambda}}(x, y)$, we have $\Theta_{H_{\lambda}}(y, 0) \subset \rho(v, y)=\rho(u, v, 0, y)$. Therefore, by cases (i)-(iv) we have $\Theta_{H_{\lambda}}(u 0, v y)=\Theta_{H_{\lambda}}(u, v) \vee \Theta_{H_{\lambda}}(0, y)$.

If $y \in X \cup\{p, q, r\}$, then $v \in Y$, by the hypotheses of case (v). But then since $u \in X$, we have $\rho_{\lambda}(u) \subseteq \rho_{\lambda}(x) \subseteq \Theta_{H_{\lambda}}(u, v)$ by Claim 1.(ii). If $y \in Y$, then $\rho_{\lambda}(u) \subseteq \rho_{\lambda}(x) \subseteq \Theta_{H_{\lambda}}(y, 0)$. In any case, we have that $\rho_{\lambda}(u) \leqq \rho_{\lambda}(x) \leqq \Theta_{H_{\lambda}}(u, v) \vee \Theta_{H_{\lambda}}(0, y)=\theta_{H_{\lambda}}(u 0, v y)$.

Suppose $\Theta_{H_{\lambda}}(u x, v y) \cong \rho_{\lambda}(x)$. We know that $u x \equiv u 0\left(\rho_{\lambda}(x)\right)$. Then we may conclude that $\Theta_{H_{\lambda}}(u, v) \vee \Theta_{H_{\lambda}}(0, y)=\Theta_{H_{\lambda}}(u 0, v y)=\rho_{\lambda}(x)=$ $\rho_{\lambda}(u, x)$. Thus we obtain $v \equiv u \equiv 0 \equiv y \equiv x\left(\rho_{\lambda}(x)\right)$. By $(\mathrm{F})$ of (\#) we obtain $\rho_{\lambda}(u, v, x, y)=\rho_{\lambda}(x)=\Theta_{H_{\lambda}}(x, y)$. This contradicts an early assumption. So we may conclude by $(\mathrm{E})$ of $(\#)$ that $\rho_{\lambda}(x) \subseteq \Theta_{H_{\lambda}}(u x, v y)$. Therefore $u 0 \equiv u x \equiv v y\left(\Theta_{H_{\lambda}}(u x, v y)\right)$.

Now we have $\Theta_{H_{\lambda}}(u x, v y)=\Theta_{H_{\lambda}}(u 0, v y) \vee \rho_{\lambda}(x)=\Theta_{H_{\lambda}}(u, v) \vee$ $\Theta_{H_{\lambda}}(0, y) \vee \rho_{\lambda}(x) \supseteqq \Theta_{H_{\lambda}}(u, v) \vee \Theta_{H_{\lambda}}(x, y) \supseteqq \Theta_{H_{\lambda}}(u x, v y)$.

This concludes the proof.

4. Ideals of a distributive lattice. This section amounts to a repeat of $\S 3$ with seemingly minor, but crucial, variations.

In this section we shall prove that the ideal lattice of every distributive lattice can be represented as the congruence lattice of a groupoid. The induction hypothesis for the proofs will include the following list of conditions. We continue the convention of $V$ meaning the join in $\langle H ; \subseteq\rangle$.

(\#) (A) $\boldsymbol{A}$ is a partial pointed groupoid.

(B) $H \cong$ Con $A$ and $\langle H$; $\leqq\rangle$ is a distributive lattice with zero.

(C) $H$ is a basis.

(D) For some nonempty $D, \operatorname{Dmn}(\cdot, \boldsymbol{A})=D \times D$.

(E) For every $a, b \in D$, it is true that $a \cdot 0 \equiv b \cdot 0(\cap H)$.

(F) For every $a \in A$ there is a $c \in D$ satisfying $c$ CLS $a$ (in $D, \bmod H)$.

(G) $A^{2}=I \cup O$ and

(i) if $\langle a, b\rangle \in O$, then $a \equiv 0\left(\Theta_{H}(a, b)\right)$, and

(ii) if $\langle y, v\rangle \in I$, then $\langle v, y\rangle \in I$, and

(iii) if $\langle y, v\rangle \in I$, then there is a $\langle d, f\rangle \in I \cap(D \times D)$ satisfying $d$ CLS $y($ in $D, \bmod H)$ and $f \operatorname{CLS} v($ in $D, \bmod H)$, and

(iv) if $c, d, e, f \in D$ and $\langle d, f\rangle \in I$, then

(a) $\langle c d, e f\rangle \in I$, and

(b) $\Theta_{H}(c d, e f)=\left[\Theta_{H}(c, e) \wedge \Theta_{H}(d, 0)\right] \vee \Theta_{H}(d, f)$,

and

(v) $\langle x, x\rangle \in I$ for every $x \in A$.

(H) For each $\Theta \in H$ there are $a, b \in A$ so that $\Theta_{H}(a, b)=\Theta$.

(I) For every $\Theta \in \operatorname{Con} A, \theta$ contains the $H$ closure of each of its finite subsets iff $\Theta$ contains the $H$ closure of each of its elements. 
Let $x, y, u, v \in A$. Set $\Phi(x y, u v)=\left[\Theta_{H}(x, u) \wedge \Theta_{H}(y, 0)\right] \vee \Theta_{H}(y, v)$. Note that $\Phi(x y, u v)$ is defined even in those cases where $x y$ or $u v$ is not defined. Let $c$ (resp., $d, e, f$ ) be a closest element in $D$ to $x$ (resp., $y, u, v)$. Set $\Psi(x y, u v)=\left(\Theta_{H}(x, c) \wedge \Theta_{H}(d, 0)\right) \vee \Theta_{H}(y, d) \vee$ $\Theta_{H}(c d, e f) \vee\left(\Theta_{H}(e, u) \wedge \Theta_{H}(f, 0)\right) \vee \Theta_{H}(f, v)=\Phi(x y, c d) \vee \Theta_{H}(c d, e f) \vee$ $\Phi(e f, u v)$.

LEMma 0. (A) If (A)-(F) of (\#) hold for $\boldsymbol{A}$ and $H$, then:

(i ) $\left[\Theta \wedge \Theta_{H}(b, 0)\right] \vee \Theta_{H}(a, b)=\left[\Theta \wedge \Theta_{H}(a, 0)\right] \vee \Theta_{H}(a, b)$ for any $\Theta \in H$ and any $a, b \in A$;

(ii) $\Theta_{H}(a b, c d) \subseteq \Phi(a b, c d)=\left[\Theta_{H}(a, c) \wedge \Theta_{H}(b, 0)\right] \vee \Theta_{H}(b, d)$ for any $a, b, c, d \in D$

(iii) For any $x, y, u, v, s, t \in A$ it is the case that $\Phi(x y, u v) \vee$ $\Phi(u v, s t) \supseteqq \Phi(x y, s t)$;

(iv) for any $x, y, u, v \in A$, it is the case that $\Psi(x y, u v)$ is independent of the choice of $c, d, e, f$.

(B) If (A)-(G) of (\#) hold for A, H, I and O, then:

(i) if $\langle y, v\rangle \in I$, then $\Phi(x y, u v) \leqq \Psi(x y, u v)$;

(ii) if $\langle y, v\rangle \in 0$, then $\Psi(x y, u v) \subseteq \Theta_{H}(y, v)=\Phi(x y, u v)$.

Proof. (A.i) holds because $\left\langle H\right.$; $\subseteq$ is distributive and $\Theta_{H}(a, 0) \vee$ $\Theta_{H}(a, b)=\Theta_{H}(b, 0) \vee \Theta_{H}(a, b)$. Note that by $(\mathrm{E})$ of $(\mathbb{H})$ we have $a b \equiv$ $a 0 \equiv c 0 \equiv c d$ under $\Theta_{H}(b, 0) \vee \Theta_{H}(b, d)$. (A.ii) now follows from the distributivity of $\langle H$; $\subseteq$. (A.iii) is a routine calculation using (A.i) and distributivity.

Now consider (A.iv). Let $c$ and $c^{\prime}$ (resp., $d$ and $d^{\prime}, e$ and $e^{\prime}, f$ and $f^{\prime}$ ) be $H$-closest elements in $D$ to $x$ (resp., $y, u, v$ ). Recall that $\Psi(x y, u v)=\Phi(x y, c d) \vee \Theta_{H}(c d, e f) \vee \Phi(e f, u v)$. We wish to show $\Psi(x y, u v)$ also $=\Phi\left(x y, c^{\prime} d^{\prime}\right) \vee \Theta_{H}\left(c^{\prime} d^{\prime}, e^{\prime} f^{\prime}\right) \vee \Phi\left(e^{\prime} f^{\prime}, u v\right)$. Note, by definition of $H$-closest that $\Theta_{H}\left(x, c^{\prime}\right)=\Theta_{H}(x, c)$, etc. So $\Phi(x y, c d)=$ $\left[\Theta_{H}(x, c) \wedge \Theta_{H}(y, 0)\right] \vee \Theta_{H}(y, d)=\left[\Theta_{H}\left(x, c^{\prime}\right) \wedge \Theta_{H}(y, 0)\right] \vee \Theta_{H}\left(y, d^{\prime}\right)=$ $\Phi\left(x y, c^{\prime} d^{\prime}\right)$, etc. Now using (A.ii) and (A.iii) we obtain $\Phi(x y, c d)=$ $\Phi(x y, c d) \vee \Phi\left(x y, c^{\prime} d^{\prime}\right)=\Phi\left(x y, c^{\prime} d^{\prime}\right) \vee \Phi\left(c^{\prime} d^{\prime}, c d\right) \supseteqq \Phi\left(x y, c^{\prime} d^{\prime}\right) \vee \Theta_{H}\left(c^{\prime} d^{\prime}\right.$, $c d) \supseteqq \Phi\left(x y, c^{\prime} d^{\prime}\right)=\Phi(x y, c d)$. That is, $\Phi(x y, c d)=\Phi\left(x y, c^{\prime} d^{\prime}\right) \vee \Theta_{H}\left(c^{\prime} d^{\prime}\right.$, $c d)$, etc. The desired result follows from this.

Let $x, y, u, v, c, d, e, f$ be as above.

Suppose $\langle y, v\rangle \in I$. Then by (G.iii) and (G.iv) of (\#) we may suppose $\Theta_{H}(c d, e f)=\Phi(c d, e f)$. Then $\Psi(x y, u v)=\Phi(x y, c d) \vee \Phi(c d, e f) \vee$ $\Phi(e f, u v) \supseteqq \Phi(x y, u v)$ by (A.iii) of this lemma.

Suppose $\langle y, v\rangle \in O$. Certainly $\Phi(x y, u v) \supseteqq \Theta_{H}(y, v)$ and $y \equiv d \equiv$ $0 \equiv f \equiv v$ under $\Theta_{H}(y, v)$. So by $(\mathrm{E})$ of (\#) we have $\Theta_{H}(c d, e f) \subseteq$ $\Theta_{H}(y, v)$. Clearly, we also have $\Phi(x y, c d)=\left[\Theta_{H}(x, c) \wedge \Theta_{H}(y, 0)\right] \vee$ $\Theta_{H}(y, d) \subseteq \Theta_{H}(y, v)$, etc. Thus $\Psi(x y, u v) \subseteq \Theta_{H}(y, v)$. 
Definition. Suppose $\langle\boldsymbol{A}, H, I, O\rangle$ satisfies (\#). $\left\langle\boldsymbol{A}^{*}, H^{*}, I^{*}, O^{*}\right\rangle$ is an extension of $\langle A, H, I, O\rangle$ iff:

(i) $\boldsymbol{A}^{*}$ is an extension of $\boldsymbol{A}$;

(ii) $A \times A \subseteq \operatorname{Dmn}\left(\cdot, A^{*}\right)$;

(iii) $I^{*} \cap A^{2}=I$ and $O^{*} \cap A^{2}=O$;

(iv) $[\Theta]_{H^{*}} \cap A^{2}=\Theta$ for any $\Theta \in H$;

(v) $H^{*}=\left\{[\Theta]_{H^{*}}: \Theta \in H\right\}$;

(vi) $\left\langle\boldsymbol{A}^{*}, H^{*}, I^{*}, O^{*}\right\rangle$ also satisfy (\#).

Note (as in §3) that it is implicit in (iv) of this definition that $[\Theta]_{H^{*}}$ is required to exist, for each $\Theta \in H$.

Lemma 1. Suppose $\langle\boldsymbol{A}, H, I, O\rangle$ satisfies (\#). If $\left\langle\boldsymbol{A}^{*}, H^{*}, I^{*}, O^{*}\right\rangle$ satisfies (i) $-(\mathrm{v})$ of the definition of "extension" (with respect to $\langle A, H, I, O\rangle$ ) and (A)-(G) of (\#), then $\left\langle A^{*}, H^{*}, I^{*}, O^{*}\right\rangle$ is an extension of $\langle A, H, I, O\rangle$.

In other words, $(\mathrm{H})$ and $(\mathrm{I})$ of (\#) are preserved "for free."

Proof. Since (H) of (\#) holds for $H$ and since (iv) and (v) of the definition hold, $H^{*}$ clearly also satisfies $(\mathrm{H})$ of (\#).

Let $\Theta$ be a congruence of $\boldsymbol{A}^{*}$ containing the $H^{*}$-closure of each of its elements. By (iv) and (v) of the definition $\left.\Theta\right|_{A} \supseteqq \Theta_{H}(a, b)$ for every $\left.\langle a, b\rangle \in \Theta\right|_{A}$. Hence $\left.\Theta\right|_{A}$ contains the $H$-closure of each of its finite subsets.

Let $X^{*}$ be a finite subset of $\Theta$. For each $p^{*} \in X^{*}$ it is the case that $\left.\left(\Theta_{H^{*}}\left(p^{*}\right)\right)\right|_{A} \in H$. So there is a $p \in A$ with $\left.\left(\Theta_{H^{*}}\left(p^{*}\right)\right)\right|_{A}=\Theta_{H}(p)$. Whence $\Theta_{H^{*}}\left(p^{*}\right)=\Theta_{H^{*}}(p)$ by (iv) and (v) of the definition. Choose and fix one such $p$ for each $p^{*}$. Let $X$ be the set of such $p$ 's. $X$ is a finite subset of $\left.\Theta\right|_{A}$ and $[X]_{H^{*}}=\left[X^{*}\right]_{H^{*}}$. By $(\mathrm{H})$ of $\left(^{*}\right)$ for $H$ and by the above, we have, for some $a, b, \Theta_{H}(a, b)=\left.[X]_{H} \cong \Theta\right|_{A}$. Hence $\langle a, b\rangle \in \Theta$ and $\Theta_{H^{*}}(a, b)=[X]_{H^{*}}$. So $\Theta \supseteqq[X]_{H^{*}}$, ending the proof.

Next we state the principal lemmas of this section.

LEMma 2. Suppose:

(0) $\left\langle A_{0}, H_{0}, I_{0}, O_{0}\right\rangle$ satisfies (\#);

(i) $\alpha$ is a limit ordinal;

(ii) for any $\beta<\gamma<\alpha\left\langle\boldsymbol{A}_{\gamma}, H_{\gamma}, I_{\gamma}, O_{\gamma}\right\rangle$ is an extension of $\left\langle\boldsymbol{A}_{\beta}, H_{\beta}\right.$, $\left.I_{\beta}, O_{\beta}\right\rangle$;

(iii) $\boldsymbol{A}_{\alpha}=\left\langle A_{\alpha}, \cdot, 0\right\rangle=\left\langle\mathbf{U}\left(A_{\beta}: \beta\langle\alpha), \cdot, 0\right\rangle\right.$

(iv) for each $\Theta \in H_{0}$ we have $\Theta_{\alpha}=\mathbf{U}\left([\Theta]_{H_{\beta}}: \beta<\alpha\right)$ and $H_{\alpha}=$ $\left\{\Theta_{\alpha}: \Theta \in H_{0}\right\}$

(v) $I_{\alpha}=\mathbf{U}\left(I_{\beta}: \beta<\alpha\right)$ and $O_{\alpha}=\mathbf{U}\left(O_{\beta}: \beta<\alpha\right)$. Then $A_{\alpha}$ is a 
pointed groupoid and $\left\langle\boldsymbol{A}_{\alpha}, H_{\alpha}, I_{\alpha}, O_{\alpha}\right\rangle$ is an extension of $\left\langle\boldsymbol{A}_{\beta}, H_{\beta}, I_{\alpha}, O_{\alpha}\right\rangle$ for all $\beta<\alpha$.

This lemma says chain unions are okay. The next lemma gives us a starting point.

LEMMA 3. If $L$ is a distributive lattice with zero, then there is $a\langle\boldsymbol{B}, H, I, O\rangle$ satisfying (\#) with $L \cong\langle H ; \subseteq\rangle$.

Suppose $\langle A, H, I, O\rangle$ satisfies (\#). For $a, b \in A[\cdot]$ and $\Theta \in H$, let $a \equiv b(\Theta\{\cdot\})$ iff one of the following holds:

(i) $a, b \in A$ and $a \equiv b(\Theta)$;

(ii) $a \in A$ and $b=u v \notin A$ and $\Theta_{H}(a, e f) \vee \Phi(e f, u v) \subseteq \Theta$, where $e$ CLS $u($ in $D, \bmod H)$ and $f$ CLS $v($ in $D, \bmod H)$;

(iii) $a \notin A$ and $b \in A$ and the condition symmetric to (ii) holds;

(iv) $a=x y \notin A$ and $b=u v \notin A$ and $\Psi(x y, u v) \cap \Phi(x y, u v) \subseteq \Theta$.

Note, as in Lemma (0.iv), the relation described in (ii) is independent of one's choice of $e, f$. Set $H\{\cdot\}=\{\Theta\{\cdot\}: \Theta \in H\}$. For $a=$ $x y \in A[\cdot]-A$, set $C(a)=\{c d: c \operatorname{CLS} x($ in $D, \bmod H)$ and $d \operatorname{CLS} y$ (in $D, \bmod H)\}$. Set $I[\cdot]=I \cup \bigcup\{\{\langle a, b\rangle,\langle b, a\rangle\}: a \in A, b \notin A$, and for some $c \in C(b)$ we have $\langle a, c\rangle \in I\} \cup\{\langle a, b\rangle: a \notin A, b \notin A$, and for some $c \in C(a)$ and $d \in C(b)$ we have $\langle c, d\rangle \in I\}$. We set $O[\cdot]=O \cup\left((A[\cdot])^{2}-\right.$ $\left.\left(A^{2} \cup I[\cdot]\right)\right)$.

LEMMA 4. If $\langle A, H, I, O\rangle$ satisfies (\#), then $H\{\cdot\}$ is a basis and $\langle\boldsymbol{A}[\cdot], H\{\cdot\}, I[\cdot], O[\cdot]\rangle$ is an extension of $\langle\boldsymbol{A}, H, I, O\rangle$.

Suppose $\langle\boldsymbol{A}, H, I, O\rangle$ satisfies (\#). Set $\left\langle\boldsymbol{A}_{0}, H_{0}, I_{0}, O_{0}\right\rangle=\langle\boldsymbol{A}, H, I, O\rangle$ and set $\left\langle\boldsymbol{A}_{n+1}, H_{n+1}, I_{n+1}, O_{n+1}\right\rangle=\left\langle\boldsymbol{A}_{n}[\cdot], H_{n}\{\cdot\}, I_{n}[\cdot], O_{n}[\cdot]\right\rangle$. Let $\left\langle\boldsymbol{A}_{\omega}\right.$, $\left.H_{\omega}, I_{\omega}, O_{\omega}\right\rangle$ be given by Lemma 2. Note that $\boldsymbol{A}_{\omega}=\boldsymbol{F r}(\boldsymbol{A})$. So we set $\boldsymbol{F r}(H)=H_{\omega}$ and $\boldsymbol{F r}(I)=I_{\omega}$ and $\boldsymbol{F r}(O)=O_{\omega}$.

Lemma 5. If $\langle A, H, I, O\rangle$ satisfies (\#), then $\langle\boldsymbol{F r}(\boldsymbol{A}), \boldsymbol{F r}(H), \boldsymbol{F r}(I)$, $\boldsymbol{F r}(O)\rangle$ is an extension of $\langle\boldsymbol{A}, H, I, O\rangle$.

Suppose that $\langle A, H, I, O\rangle$ satisfies (\#) and $\operatorname{Dmn}(\cdot, A)=A^{2}$. Also suppose $\lambda=\langle a, b, c, d\rangle \in A^{4}$ and $a \neq \equiv b(\cap H)$ and $c \equiv d\left(\Theta_{H}(a, b)\right)$. Take $p, q, r \notin A$ and set $\boldsymbol{A}^{\prime}=\langle A \cup\{p, q, r\}, \cdot, 0\rangle$ where $x \cdot y$ is defined (and equal $x \cdot y$ in $A$ ) iff $x, y \in A$. For $\Theta \in H$ for which it is not the case that $a \equiv b \equiv c \equiv 0(\Theta)$ set $\Theta^{\prime}=\Theta \cup\{\langle p, p\rangle,\langle q, q\rangle,\langle r, r\rangle\}$. For $\Theta \in H$ with $a \equiv b \equiv c \equiv 0(\Theta)$ set $\Theta^{\prime}=\Theta \cup\left(0 / \Theta \cup\{p, q, r\}^{2}\right)$. Finally set $H^{\prime}=$ $\left\{\Theta^{\prime}: \Theta \in H\right\}$ and $I^{\prime}=I \cup\{\langle p, p\rangle,\langle q, q\rangle,\langle r, r\rangle\}$ and $O^{\prime}=O \cup\left(\left(A^{\prime}\right)^{2}-\right.$ $\left.\left(I^{\prime} \cup A^{2}\right)\right)$. Let $\Phi$ be the smallest equivalence relation on $A^{\prime}[\cdot]$ which includes $\langle c, a p\rangle$ and $\langle b p, b q\rangle$ and $\langle a q, a r\rangle$ and $\langle b r, d\rangle$. $\Phi$ is a con- 
gruence relation of $A^{\prime}[\cdot]$ because $\left.\Phi\right|_{A^{\prime}}$ is the equality relation (this is because $a \neq b)$ and $\operatorname{Dmn}\left(\cdot, \boldsymbol{A}^{\prime}[\cdot]\right)=\left(A^{\prime}\right)^{2}$. Set $\boldsymbol{A}_{\lambda}=\left(\boldsymbol{A}^{\prime}[\cdot]\right) / \Phi=$ $\left\langle A_{\lambda}, \cdot, 0\right\rangle$. Since $\left.\Phi\right|_{A^{\prime}}=\Delta, \boldsymbol{A}_{\lambda}$ is an extension of $\boldsymbol{A}^{\prime}$. So we assume $A^{\prime} \subseteq A_{\lambda}$. For each $\Theta \in H$, let $\Theta_{\lambda}$ be $\left(\Theta^{\prime}\{\cdot\}+\Phi\right) / \Phi$ where + represents equivalence relation join. Let $T=p A^{\prime} \cup q A^{\prime} \cup r A^{\prime} \cup\{p, q, r\}$. Let $\langle u, v\rangle \in I_{\lambda}$ iff one of the following holds:

(i ) $\langle u, v\rangle=\langle x / \Phi, y \mid \Phi\rangle$ with $\langle x, y\rangle \in I^{\prime}[\cdot]$ and $x, y \in A^{\prime} \cup T$;

(ii) $\langle u, v\rangle \in\left(A p^{\prime}\right)^{2} \cup\left(A q^{\prime}\right)^{2} \cup\left(A r^{\prime}\right)^{2}$;

(iii) $\langle u, v\rangle \in\left(A p^{\prime} \times A q^{\prime}\right) \cup\left(A q^{\prime} \times A r^{\prime}\right)$;

(iv) $\langle u, c\rangle$ satisfies (i) and $v \in A p^{\prime} \cup A q^{\prime}$;

(v) $\langle u, d\rangle$ satisfies (i) and $v \in A q^{\prime} \cup A r^{\prime}$;

(vi) $\langle c, d\rangle \in I$ and $u \in A r^{\prime}$ and $v \in A p^{\prime}$;

(vii) $\langle v, u\rangle$ satisfies one of (iii)-(vi).

Let $O_{\lambda}=O^{\prime} \cup\left(A_{\lambda}^{2}-\left(\left(A^{\prime}\right)^{2} \cup I_{\lambda}\right)\right)$.

LEMMA 6. Under the above hypotheses the following hold:

(i) $\left\langle\boldsymbol{A}^{\prime}, H^{\prime}, I^{\prime}, O^{\prime}\right\rangle$ is an extension of $\langle\boldsymbol{A}, H, I, O\rangle$;

(ii) $\left\langle\boldsymbol{A}_{\lambda}, H_{\lambda}, I_{\lambda}, O_{\lambda}\right\rangle$ is an extension of $\langle\boldsymbol{A}, H, I, O\rangle$;

(iii) in $\boldsymbol{A}_{\lambda}$ we have $c \equiv d(\Theta(a, b))$.

The last part of the above lemma means that if $\Psi \in \operatorname{Con}\left(\boldsymbol{A}_{\lambda}\right)$ and $a \equiv b(\Psi)$, then also $c \equiv d(\Psi)$.

We will prove these lemmas later.

THEOREM 3. If $L$ is a distributive lattice, then there is a pointed groupoid $\boldsymbol{A}$ satisfying the following:

(i) $\operatorname{Con}(\boldsymbol{A})$ is isomorphic to the lattice of ideals of $L$;

(ii) if $c \equiv d(\Theta(a, b))$, then there are $p, q, r \in A$ so that $c=a p$ and $b p=b q$ and $a q=a r$ and $b r=d$;

(iii) all joins in Con $\boldsymbol{A}$ are type-3.

We shall prove this theorem assuming Lemmas 2-6. As in $\S 3$, we can reduce our notational complexity by first proving another lemma.

Suppose $\boldsymbol{A}$ is a pointed groupoid and $\langle\boldsymbol{A}, H, I, O\rangle$ satisfies (\#). Index $\left\{\lambda: \lambda=\langle a, b, c, d\rangle \in A^{4}, a \neq \equiv(\bigcap H), c \equiv d\left(\Theta_{H}(a, b)\right)\right\}$ by its cardinal number $\kappa$. Set $\langle\boldsymbol{A}, H, I, O\rangle=\left\langle\boldsymbol{A}_{0}, H_{0}, I_{0}, O_{0}\right\rangle$. Suppose $\alpha \leqq \kappa$ and $\left\langle\boldsymbol{A}_{\beta}, H_{\beta}, I_{\beta}, O_{\beta}\right\rangle$ has been defined for all $\beta<\alpha$ and for $\gamma<\beta<\alpha$ that $\left\langle\boldsymbol{A}_{\beta}, H_{\beta}, I_{\beta}, O_{\beta}\right\rangle$ is an extension of $\left\langle\boldsymbol{A}_{\gamma}, H_{\gamma}, I_{r}, O_{\gamma}\right\rangle$ and $\boldsymbol{A}_{\beta}$ is a pointed groupoid for all $\beta<\alpha$. If $\alpha=\beta+1$, set $\left\langle\boldsymbol{A}_{\alpha}, H_{\alpha}, I_{\alpha}, O_{\alpha}\right\rangle=$ $\left\langle\boldsymbol{F r}\left(\left(\boldsymbol{A}_{\beta}\right)_{\lambda_{\beta}}\right), \boldsymbol{F r}\left(\left(H_{\beta}\right)_{\lambda_{\beta}}\right), \boldsymbol{F r}\left(\left(I_{\beta}\right)_{\lambda_{\beta}}\right), \boldsymbol{F r}\left(\left(O_{\beta}\right)_{\lambda_{\beta}}\right)\right\rangle$. If $\alpha$ is a limit ordinal, then we let $\left\langle\boldsymbol{A}_{\alpha}, H_{\alpha}, I_{\alpha}, O_{\alpha}\right\rangle$ be given by Lemma 2. Set $\left\langle\boldsymbol{A}^{\prime \prime}, H^{\prime \prime}\right.$, $\left.I^{\prime \prime}, O^{\prime \prime}\right\rangle=\left\langle\boldsymbol{A}_{\kappa}, H_{\kappa}, I_{\kappa}, O_{\kappa}\right\rangle$. 
LEMMA 7. Under the above hypotheses the following hold:

(i) $\boldsymbol{A}^{\prime \prime}$ is a pointed groupoid;

(ii) $\left\langle\boldsymbol{A}^{\prime \prime}, H^{\prime \prime}, I^{\prime \prime}, O^{\prime \prime}\right\rangle$ is an extension of $\langle A, H, I, O\rangle$;

(iii) if $a, b \in A$ and $a \neq \equiv(\cap H)$ and $c \equiv d\left(\Theta_{H}(a, b)\right)$; then there are $p, q, r \in A^{\prime \prime}$ so that $c=a p$ and $b q=b q$ and $a q=a r$ and $b r=d$.

(iv) if $a, b \in A$ and $a \neq \equiv(\cap H)$, then in $A^{\prime \prime}$ we have $\Theta(a, b) \supseteqq$ $\theta_{H}(a, b)$.

REMARK. The "remark" after Lemma 9 of $\S 3$, after obvious trivial changes, applies here as well.

Proof. Using the remark and Lemmas 2, 5, and 6 and transfinite induction, one can easily show for each $\alpha \leqq \kappa$ that $\left\langle\boldsymbol{A}_{\alpha}, H_{\alpha}, I_{\alpha}, O_{\alpha}\right\rangle$ exists and is an extension, for each $\beta<\alpha$, of $\left\langle\boldsymbol{A}_{\beta}, H_{\beta}, I_{\beta}, O_{\beta}\right\rangle$ and that $\boldsymbol{A}_{\alpha}$ is a (total) pointed groupoid. Thus (i) and (ii) hold.

Let $a, b \in A$, and let $c \equiv d\left(\Theta_{H}(a, b)\right)$. Suppose $a \not \equiv b(\cap H)$. Then $\langle a, b, c, d\rangle$ is some $\lambda_{\beta}$, and we have the required $p, q, r$. Thus (iii) holds. (iv) follows easily from (iii).

Proof of Theorem 3. Let $L$ be a distributive lattice. We may suppose $L$ has a zero. Set $\left\langle\boldsymbol{A}_{0}, H_{0}, I_{0}, O_{0}\right\rangle=\langle\boldsymbol{F r}(B), \boldsymbol{F r}(H), \boldsymbol{F r}(I), \boldsymbol{F r}(O)\rangle$ where $\langle\boldsymbol{B}, H, I, O\rangle$ is given by Lemma 3. Set $\left\langle\boldsymbol{A}_{n+1}, H_{n+1}, I_{n+1}, O_{n+1}\right\rangle=$ $\left\langle\boldsymbol{A}_{n}^{\prime \prime}, H_{n}^{\prime \prime}, I_{n}^{\prime \prime}, O_{n}^{\prime \prime}\right\rangle$ using the construction for Lemma 7 . Consider $\left\langle\boldsymbol{A}_{\omega}, H_{\omega}, I_{\omega}, O_{\omega}\right\rangle$ as given by Lemma 2. By Lemma 2, $\boldsymbol{A}_{\omega}$ is a (total) pointed groupoid. Lemma 3, Lemma 5, Lemma 7, Lemma 2, induction, the transitivity of the extension relation, and the remark after Lemma 7 all imply that $\left\langle H_{\omega} ; \cong\right\rangle \cong\langle H ; \cong\rangle \cong L$.

Claim 1. If $a \neq \equiv b\left(\bigcap H_{\omega}\right)$, then $\Theta(a, b)=\Theta_{H_{\omega}}(a, b)$.

We suppose $a \neq \equiv b\left(\cap H_{\omega}\right)$. By Lemma 7 , Lemma 2 , and the "remark" after Lemma 7 we have $a \not \equiv b\left(\cap H_{n}\right)$ for any $n$ satisfying $a, b \in A_{n}$. If $a, b \in A_{n}$, we let $\Theta_{n}(a, b)$ denote the smallest congruence relation of $\boldsymbol{A}_{n}$ containing $\langle a, b\rangle$. By (iv) of Lemma 7 and induction, by Lemma 7 and Lemma 2 and the remark after Lemma 7, and from general principals we have that $\Theta_{H_{\omega}}(a, b) \supseteqq \Theta(a, b) \supseteqq \mathbf{U}\left(\Theta_{n+1}(a, b)\right.$ : $\left.a, b \in A_{n}\right) \supseteqq \mathbf{U}\left(\Theta_{H_{n}}(a, b): a, b \in A_{n}\right)=\Theta_{H_{\omega}}(a, b)$. This ends the proof of Claim 1.

Let $M$ be the filter (dual ideal) of Con $\boldsymbol{A}_{\omega}$ consisting of all congruences containing $\left(\bigcap H_{\omega}\right)$.

Claim 2. If $\Theta \in M$, then $\Theta$ contains the $H_{\omega}$-closure of each of its finite subsets.

By hypothesis and Claim $1 \Theta \supseteq \Theta_{H_{\omega}}(a, b)$ for each $\langle a, b\rangle \in \Theta$. Now (I) of (\#) finishes the proof of the claim. 
$M$ is an algebraic closure system. Let $K=\left\{[X]_{M}: X\right.$ is a finite subset of $\left.A_{\omega}^{2}\right\}$.

\section{Claim 3. $K=H_{\omega}$.}

$K$ and $H_{\omega}$ are both bases. So it suffices to show that $[X]_{M}=$ $[X]_{H_{\omega}}$ for each finite subset $X$ of $A_{\omega}^{2}$. Let $X$ be such. By Claim 2 and general principles we have $[X]_{K}=[X]_{M} \supseteq[X]_{H_{\omega}} \supseteq[X]_{\operatorname{Con} A_{\omega}}+$ $\left(\cap H_{\omega}\right)=[X]_{M}=[X]_{K}$, where + is equivalence relation join. This establishes the claim.

Set $\boldsymbol{A}=\boldsymbol{A} \omega /\left(\cap H_{\omega}\right)$. Since $\langle K ; \cong\rangle=\left\langle H_{\omega} ; \cong\right\rangle \cong L$, it follows (see $\S 2$ ) that Con $\boldsymbol{A} \cong\langle M ; \cong\rangle \cong$ the ideal lattice of $L$. This establishes (i) of the theorem.

Suppose $c \equiv d(\Theta(a, b))$ in $\boldsymbol{A}$.

Suppose that $a \neq b$. Let $a^{\prime}, b^{\prime}, c^{\prime}, d^{\prime} \in A_{\omega}$ be such that $a^{\prime} /\left(\cap H_{\omega}\right)=$ $a$, etc. Certainly $a^{\prime} \neq b^{\prime}\left(\cap H_{\omega}\right)$. By general principles and the above claims, we obtain $c^{\prime} \equiv d^{\prime}\left(\Theta_{H_{\omega}}\left(a^{\prime}, b^{\prime}\right)\right)$. Now Lemma 2, Lemma 7 and "remark" after Lemma 7 yields $p^{\prime}, q^{\prime}, r^{\prime}$ satisfying $a^{\prime} p^{\prime}=c$, etc. With $p=p^{\prime} /\left(\cap H_{\omega}\right)$, etc., we have $a p=c, b p=b q$, etc.

Now suppose $a=b$. Then certainly $c=d$. If $\boldsymbol{A}$ is the one element algebra, $p=q=r=0=$ the one element, will do. If $\boldsymbol{A}$ is not the one element algebra, choose some $b^{*} \neq b$. Then $c \equiv d\left(\Theta\left(a, b^{*}\right)\right)$. By the previous case there are $p^{*}, q^{*}, r^{*}$ with $a p^{*}=c, b^{*} p^{*}=b^{*} q^{*}$, etc. With $p=q=r=p^{*}$ we have $c=d=a p=b p=b q=a q=$ $a r=b r=d$.

Thus (ii) of the theorem is true. We defer the proof of (iii) till after the proof of Lemma 3.

Proof of Lemma 3. We suppose $L$ is a distributive lattice with zero. Set $B=L$, and let 0 be the zero of $L$. Set $0 \cdot 0=0$ and $\operatorname{Dmn}(\cdot)=\{\langle 0,0\rangle\}$ and $\boldsymbol{B}=\langle B, \cdot, 0\rangle$. For $b \in B$ define $\Theta_{b}$ by $x \equiv y\left(\Theta_{b}\right)$ iff $x=y$ or $x \vee y \leqq b$. We set $H=\left\{\Theta_{b}: b \in B\right\}$ and $I=B^{2}$ and $O=$ $\varnothing$. It is not too hard to show that $\langle\boldsymbol{B}, H, I, O\rangle$ has the required properties. Details are left to the reader.

Proof of (iii) of Theorem 3. For the $B$ and $H$ of the proof of Lemma 3, we have $\cap H=\Delta$, and so $\left.\left(\cap H_{\omega}\right)\right|_{B}=\Delta$. Thus we may suppose $B \cong A$. Let $c, d \in A$ and $\Theta, \Phi \in \operatorname{Con}(\boldsymbol{A})$. Suppose $c \equiv d(\Theta \vee \Phi)$ and $c \not \equiv d(\Theta)$ and $c \not \equiv d(\Phi)$. Since $\Theta(c, d)$ is compact in $\operatorname{Con}(\boldsymbol{A})$, we can find compact $\Theta_{0}, \Phi_{0}$ such that $c \equiv d\left(\Theta_{0} \vee \Phi_{0}\right)$ and $\Theta_{0} \leqq \Theta$ and $\Phi_{0} \subseteq \Phi$. Let $\Theta_{0}^{\prime}$ and $\Phi_{0}^{\prime}$ be the congruences of $\boldsymbol{A}_{\omega}$ satisfying $\Theta_{0}^{\prime} /\left(\bigcap H_{\omega}\right)=\Theta_{0}$ and $\Phi_{0}^{\prime} /\left(\bigcap H_{\omega}\right)=\Phi_{0} . \quad \Theta_{0}^{\prime}$ and $\Phi_{0}^{\prime}$ are in $H_{\omega}$; also neither equals $\left(\cap H_{\omega}\right)$. So $\left.\Theta_{0}^{\prime}\right|_{B} \in H$ and $\left.\Phi_{0}^{\prime}\right|_{B} \in H$ and neither equals $\triangle$. So there exist nonzero $a, b \in B$ with $\Theta_{H}(a, 0)=\left.\Theta_{0}^{\prime}\right|_{B}$ and $\Theta_{H}(b, 0)=\left.\Phi_{0}^{\prime}\right|_{B}$ 
and $\Theta_{H}(a, b)=\left.\left.\Theta_{0}^{\prime}\right|_{B} \vee \Phi_{0}^{\prime}\right|_{B}$. Hence $\Theta_{H_{\omega}}(a, 0)=\Theta_{0}^{\prime}$ and $\Theta_{H_{\omega}}(b, 0)=\Phi_{0}^{\prime}$ and $\Theta_{H_{\omega}}(a, b)=\Theta_{0}^{\prime} \vee \Phi_{0}^{\prime}$. We have now that $\Theta(a, 0)=\Theta_{0}$ and $\Theta(b, 0)=\Phi_{0}$ and $\Theta(a, b)=\Theta_{0} \vee \Phi_{0}$. The rest is as in the proof of Theorem 2, ending our proof of Theorem 3 .

Proof of Lemma 2. Suppose $x, y \in A_{\alpha}$. Then there is a $\beta<\alpha$ with $x, y \in A_{\beta}$. By (ii) of the definition of extension, $x \cdot y$ is defined in $\boldsymbol{A}_{\beta+1}$ and, hence, in $\boldsymbol{A}_{\alpha}$. Thus $\boldsymbol{A}_{\alpha}$ is a (total) pointed groupoid.

Since $A_{\alpha} \times A_{\alpha}=\operatorname{Dmn}\left(\cdot, A_{\alpha}\right)$, (i)-(v) of the definition of extension and (D) and (F) of (\#) are obvious. Establish that $H_{\alpha}$ is a basis as in the proof of Lemma 3.6, yielding (C) of (\#). (A) and (B) of (\#) are obvious. Clearly $\cap H_{\alpha}=\left(\cap H_{0}\right)_{\alpha}=\bigcup\left(\left[\cap H_{0}\right]_{H_{\beta}}: \beta<\alpha\right)$. So (E) of (\#) for $\boldsymbol{A}_{\alpha}$ and $H_{\alpha}$ follows. Let $a, b \in A_{\alpha}$. Pick any $\beta<\alpha$ such that $a, b \in A_{\beta}$. By the definition of extension (as applied to $\left\langle\boldsymbol{A}_{\beta}, \cdots\right\rangle$ and $\left\langle\boldsymbol{A}_{\gamma}, \cdots\right\rangle$ for another $\gamma\langle\alpha$ ) and the "remark" after Lemma 7, it is clearly the case that $\Theta_{H_{\alpha}}(a, b)=\left(\left.\left(\Theta_{H_{\beta}}(a, b)\right)\right|_{A_{0}}\right)_{\alpha}$. (G) of (\#) for $\left\langle\boldsymbol{A}_{\alpha}, H_{\alpha}, I_{\alpha}, O_{\alpha}\right\rangle$ now follows easily from the hypotheses and the definition of extension and from the fact that " $D$ " $=A_{\alpha}$. So by Lemma 1, we are done with the proof of Lemma 2.

Here, finally, is the proof of one of the two crucial lemmas.

Proof of Lemma 4. The first thing we need to establish is that each $\Theta\{\cdot\}$ is a congruence relation on $\boldsymbol{A}[\cdot]$. Since, by Lemma 0 , $\Psi(x y, u v)=\Psi(u v, x y)$ and $\Phi(x y, u v)=\Phi(u v, x y)$, we only need to show that each $\Theta\{\cdot\}$ is transitive. Let $\Theta \in H$, and suppose $a \equiv b(\Theta\{\cdot\})$ and $b \equiv c(\Theta\{\cdot\})$. The only cases we need to consider are:

(a) $a \in A, b \in A, c \notin A$;

(b) $a \in A, b \notin A, c \in A$;

(c) $a \notin A, b \in A, c \notin A$;

(d) $a \in A, b \notin A, c \notin A$;

(e) $a \notin A, b \notin A, c \notin A$.

In what follows, "cd $\in C(x y)$ " will abbreviate " $c d$ is an element of $C(x y)$ and $c \operatorname{CLS} x($ in $D, \bmod H)$ and $d \operatorname{CLS} y($ in $D, \bmod H)$."

Case a. $c=r s \notin A$. For any $h i \in C(r s)$ the definition of $\Theta\{\cdot\}$ yields $a \equiv b \equiv h i(\Theta)$ and $\Phi(h i, r s) \subseteq \Theta$. Thus $a \equiv h i(\Theta)$ and $a \equiv c(\Theta\{\cdot\})$.

Case b. $b=u v \notin A$. For any $f g \in C(u v)$ the definition of $\Theta\{\cdot\}$ yields $a \equiv f g \equiv c(\Theta)$ and $a \equiv c(\Theta)$.

Case c. $\quad a=x y \notin A$ and $c=r s \notin A$. For any $d e \in C(x y)$ and $h i \epsilon$ $C(r s)$, the definition of $\Theta\{\cdot\}$ yields $\Phi(x y, d e) \subseteq \Theta$ and $d e \equiv b \equiv h i(\Theta)$ and $\Phi(h i, r s) \cong \Theta$. Hence $\Psi(x y, r s) \cong \Theta$ and $a \equiv c(\Theta\{\cdot\})$. 
Case d. $\quad b=u v \notin A$ and $c=r s \notin A$. Let $f g \in C(u v)$ and $h i \in C(r s)$. By definition of $\Theta\{\cdot\}$ we have $a \equiv f g(\Theta)$ and $\Phi(f g, u v) \leqq \Theta$. Suppose $\langle v, s\rangle \in O$. By Lemma 0.B.(ii) and the definition of $\Theta\{\cdot\}, \Psi(u v, r s) \subseteq \Theta$. But then $f g \equiv h i(\Theta)$ and $\Phi(h i, r s) \cong \Theta$. Transitivity of $\Theta$ yields $a \equiv$ $h i(\Theta)$. Hence $a \equiv c(\Theta\{\cdot\})$. Suppose $\langle v, s\rangle \in I$. By Lemma 0.B.(i) and the definition of $\Theta\{\cdot\}, \Phi(u v, r s) \cong \Theta$. By Lemma 0.A.(iii) $\Theta \supseteqq \Phi(r s, f g)=$ $\left[\Theta_{H}(r, f) \wedge \Theta_{H}(s, 0)\right] \vee \Theta_{H}(s, g)$. Since $f, g \in D$ and $h$ and $i$ are the closest things to $r$ and $s$, respectively, we have $\Theta_{H}(r, f) \supseteqq \Theta_{H}(r, h)$ and $\Theta_{H}(s, g) \supseteqq \Theta_{H}(s, i)$. It follows that $\Theta \supseteqq \Phi(r s, f g) \supseteqq \Phi(r s, h i)$. Using 0 .A.(iii) again and 0.A.(ii) we have $\Theta_{H}(f g, h i) \subseteq \Phi(f g, h i) \subseteq \Theta$. That is $a \equiv f g \equiv h i(\Theta)$. By transitivity of $\Theta, a \equiv h i(\Theta)$. Just above we have $\Phi(r s, h i) \cong \Theta$. By definition, $a \equiv c(\Theta\{\cdot\})$. (G) of (\#) says there are no more subcases.

Case e. $\quad a=x y \notin A$ and $b=u v \notin A$ and $c=r s \notin A$. By Lemma 0. A. (iii) and symmetry, the only subcase we need consider is the one in which $\langle y, v\rangle \in O$. Let $d e \in C(x y)$ and $f g \in C(u v)$. Consider $\Psi(x y, u v)$, which is contained in $\Theta$ by 0 .B.(ii). Then by definition and hypothesis, $a \equiv d e(\Theta\{\cdot\})$ and $d e \equiv b(\Theta\{\cdot\})$ and $b \equiv c(\Theta\{\cdot\})$. Then Case (c) yields $d e \equiv c(\Theta\{\cdot\})$, and Case (b) yields $a \equiv c(\Theta\{\cdot\})$.

It is now clear that (i)-(v) of the definition of extension and (A), (B), (D) of (\#) hold.

The definition of $\Theta\{\cdot\}$ for each $\Theta \in H$ makes it obvious that $[\langle a, b\rangle]_{H \nmid \cdot\}}\left(=\Theta_{H\{\cdot \cdot(}(a, b)\right)$ exists for each $a, b \in A[\cdot]$. For example, if $a \in A$ and $b=u v \notin A$ and $e f \in C(u v)$, then $\Theta_{H(\cdot \cdot)}(a, b)=\left[\Theta_{H}(a, e f) \vee \Phi(e f, u v)\right]\{\cdot\}$. Now apply Lemma 2.2 to $H$ and then to $H\{\cdot\}$, concluding that $H\{\cdot\}$ is a basis. So $(\mathrm{C})$ of (\#) holds.

It is clear that for any $a, b \in A$ we have $\Phi(a 0, b 0)=\bigcap H$. If both $a, b \in D$, then by 0 .A.(ii), we have $\langle a 0, b 0\rangle \in \Theta_{H}(a 0, b 0) \leqq$ $\Phi(a 0, b 0)=\bigcap H \leqq \bigcap(H\{\cdot\})$. If neither $a$ nor $b$ is in $D$, we have $\langle a 0, b 0\rangle \in \Theta_{H(\cdot)}(a 0, b 0) \leqq(\Phi(a 0, b 0))\{\cdot\}=(\bigcap H)\{\cdot\}=\bigcap(H\{\cdot\})$. Suppose $a \in D$ and $b \notin D$. There is a $c 0 \in C(b 0)$. Now $\Theta_{H|\cdot|}(a 0, b 0)=(\Phi(a 0, c 0) \vee$ $\Phi(c 0, b 0))\{\cdot\}=(\bigcap H)\{\cdot\}=\bigcap(H\{\cdot\})$. So $(\mathrm{E})$ of (\#) holds.

Clearly any $c \in C(a)$ satisfies $c \operatorname{CLS} a$ (in $A, \bmod H\{\cdot\})$ for any $a \in A[\cdot]-A$. And we obtain (F) of (\#).

Consider $(\mathrm{G})$ of (\#). Clearly $I[\cdot] \cup O[\cdot]=(A[\cdot])^{2}$.

Suppose $\langle a, b\rangle \in O[\cdot]$. If $\langle a, b\rangle \in O$, then $a \equiv 0\left(\Theta_{H}(a, b)\right)$. So certainly $a \equiv 0\left(\Theta_{H(\cdot)}(a, b)\right)$. Suppose $a \in A$ and $b \notin A$. Then $\langle a, b\rangle \notin$ $I[\cdot]$. So for every $c \in C(b)$ we have $\langle a, c\rangle \notin I$. Thus $\langle a, c\rangle \in O$ for every such $c$, and $a \equiv 0\left(\Theta_{H}(a, c)\right)$. But $\Theta_{H}(a, c) \leqq \Theta_{H \cdot \cdot l}(a, b)$. Suppose $a \notin A$ and $b \notin A$. Then for every $c d \in C(a)$ and $e f \in C(b)$ we have $\langle c d, e f\rangle \notin I$. Hence $\langle d, f\rangle \notin I$ and $c d \equiv 0\left(\Theta_{H}(c d, e f)\right)$ and $\Theta_{H(\cdot)}(a, b)=$ $(\Psi(a, b))\{\cdot\}$ - This makes sense because $a$ and $b$ have unique factorizations - and so $a \equiv c d \equiv e f \equiv b\left(\Theta_{H \mid \cdot\}}(a, b)\right)$, and then $a \equiv c d \equiv$ 
$0\left(\Theta_{H(\cdot)}(a, b)\right)$. So (i) of $(\mathrm{G})$ of (\#) holds.

(ii) and (iii) of $(G)$ of $(\#)$ clearly are satisfied (just look at the definition of $I[\cdot])$.

Let $c, d, e, f \in A$ with $\langle d, f\rangle \in I[\cdot]$. It follows that $\langle d, f\rangle \in I$. If $c d, e f \in A$, then $\langle c d, e f\rangle \in I \subseteq I[\cdot]$. Suppose $c d \in A$ and $e f \notin A$. By G.(iii) of (\#) for $I$ we may choose $g h \in C(e f)$ with $\langle d, h\rangle \in I$. By G.(iv)(a) of (\#) for $I$ we have $\langle c d, g h\rangle \in I$, and so $\langle c d, e f\rangle \in I[\cdot]$. Suppose $c d \notin A$ and $e f \notin A$. Similar to the preceding case, we can find $\langle g h, i j\rangle \in I \cap(C(c d) \times C(e f))$, and then $\langle c d, e f\rangle \in I[\cdot]$. Thus G.(iv)(a) of (\#) holds for $I[\cdot]$.

Let us continue with the same $c, d, e, f . \quad \Phi^{*}(x y, u v)$ will represent $\left[\Theta_{H \nmid \cdot\}}(x, u) \wedge \Theta_{H \nmid \cdot \mid}(y, 0)\right] \vee \Theta_{H \mid \cdot \cdot}(y, v)$, while $\Phi(x y, u v)$ still equals $\left[\Theta_{H}(x, u) \wedge\right.$ $\left.\Theta_{H}(y, 0)\right] \vee \Theta_{H}(y, v)$. If both $c d$, ef are in $A$ or both are not in $A$,

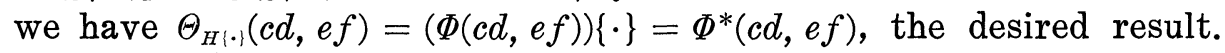
Suppose then that $c d \in A$ and $e f \notin A$. We may choose $g h \in C(e f)$ with $\langle d, h\rangle \in I$, and then $\Theta_{H \cdot \cdot \cdot\}}(c d, e f)=\left[\Theta_{H}(c d, g h) \vee \Phi(g h, e f)\right]\{\cdot\}=[\Phi(c d, g h) \vee$ $\Phi(g h, e f)]\{\cdot\} \supseteqq[\Phi(c d, e f)]\{\cdot\}=\Phi^{*}(c d, e f) \supseteqq \Theta_{H \cdot \cdot \cdot}(c d, e f)$, where the last inequality is supplied by (ii) of (A) of Lemma 0 (note that (A) of the lemma only requires (A)-(F) of (\#)). Thus G.(iv)(b) of (\#) holds for $I[\cdot]$.

Clearly, (v) of $(\mathrm{G})$ of $(\#)$ holds for $I[\cdot]$.

By Lemma 1, we are done.

Proof of Lemma 5. This follows from Lemma 4, the transitivity of the extension relation, and Lemma 2.

Now we come to the proof of the last (and second crucial) lemma.

Proof of Lemma 6. First we show that $\left\langle\boldsymbol{A}^{\prime}, H^{\prime}, I^{\prime}, O^{\prime}\right\rangle$ is an extension of $\langle A, H, I, O\rangle$.

That (i)-(v) of the definition of extension and (A), (B), (D) of (\#) hold is obvious. If $x, y \in H$, then $\Theta_{H}(x, y)=\left(\Theta_{H}(x, y)\right)^{\prime}$. If $x \neq y$ and $x, y \in\{p, q, r\}$, then $\Theta_{H^{\prime}}(x, y)=\left(\Theta_{H}(a, b, c, 0)\right)^{\prime}$. If $x \in A$ and $y \in\{p, q, r\}$, then $\Theta_{H^{\prime}}(x, y)=\left(\Theta_{H}(a, b, c, 0) \vee \Theta_{H}(0, x)\right)^{\prime}$. Apply Lemma 2.2 to $H$, use the above and $H^{\prime}=\left\{\Theta^{\prime}: \Theta \in H\right\}$, apply Lemma 2.2 to $H^{\prime}$, and conclude that $H^{\prime}$ is a basis. Whence (C) of (\#) holds. For every $a, b \in A$ we know $\langle a \cdot 0, b \cdot 0\rangle \in \bigcap H \subseteq(\bigcap H)^{\prime}=\bigcap\left(H^{\prime}\right)$. That is, (E) of (\#) is valid. For any $x \in[p, q, r] 0 \operatorname{CLS} x$ (in $A$, mod $H^{\prime}$ ) obviously holds (see the description of $\Theta_{H^{\prime}}(x, y)$ ). (F) of (\#) follows.

Clearly $\left(A^{\prime}\right)^{2}=I^{\prime} \cup O^{\prime}$. (iii) and (iv) of (G) of (\#) follow from the hypothesis and construction. (i) also follows immediately from the construction and hypothesis. Consider (ii). If $\langle y, v\rangle \in I^{\prime}-I$, then $\langle 0,0\rangle$ will do for the required $\langle d, f\rangle$. Otherwise, $\langle d, f\rangle=\langle y, v\rangle$ will suffice.

Now (i) of this lemma follows from Lemma 1. 
Next we will show that $\left\langle A_{\lambda}, H_{\lambda}, I_{\lambda}, O_{\lambda}\right\rangle$ is an extension of $\left\langle\boldsymbol{A}^{\prime}, H^{\prime}, I^{\prime}, O^{\prime}\right\rangle$. Then (ii) of this lemma will follow from the transitivity of the extension relation.

Since $\Theta_{\lambda}=\left(\Theta^{\prime}\{\cdot\}+\Phi\right) / \Phi$, we will need a good description of $\Theta^{\prime}\{\cdot\}+$ $\Phi$ in order that we may proceed. The definition of $T$ is before that of $I_{\lambda}$.

Claim 1. Let $\Theta \in H$.

(i) If $a \equiv b \equiv c \equiv 0(\Theta)$, then $\Phi \leqq \Theta^{\prime}\{\cdot\}$. Moreover, $(\{0, c, d\} \cup$ $A p \cup A q \cup A r)^{2} \subseteq \Theta^{\prime}\{\cdot\}$.

(ii) If $x, y \in A^{\prime} \cup T$, then $x \equiv y\left(\Theta^{\prime}\{\cdot\}+\Phi\right)$ iff $x \equiv y\left(\Theta^{\prime}\{\cdot\}\right)$.

(iii) If $\langle x, y\rangle \in\left(A^{\prime} p\right)^{2} \cup\left(A^{\prime} q\right)^{2} \cup\left(A^{\prime} r\right)^{2}$, then $x \equiv y\left(\Theta^{\prime}\{\cdot\}+\Phi\right)$ iff $x \equiv y\left(\Theta^{\prime}\{\cdot\}\right)$.

(iv) If $\langle x, y\rangle \in A^{\prime} p \times A^{\prime} q$, then $x \equiv y\left(\Theta^{\prime}\{\cdot\}+\Phi\right)$ iff $x \equiv b p\left(\Theta^{\prime}\{\cdot\}\right)$ and $b q \equiv y\left(\Theta^{\prime}\{\cdot\}\right)$.

( v ) If $\langle x, y\rangle \in A^{\prime} q \times A^{\prime} r$, then $x \equiv y\left(\Theta^{\prime}\{\cdot\}+\Phi\right)$ iff $x \equiv a q\left(\Theta^{\prime}\{\cdot\}\right)$ and $a r \equiv y\left(\Theta^{\prime}\{\cdot\}\right)$.

(vi) If $x \in A^{\prime} \cup T$ and $y \in A^{\prime} p \cup A^{\prime} q$, then $x \equiv y\left(\Theta^{\prime}\{\cdot\}+\Phi\right)$ iff $x \equiv$ $c\left(\Theta^{\prime}\{\cdot\}\right)$ and $a p \equiv y\left(\Theta^{\prime}\{\cdot\}+\Phi\right)$.

(vii) If $x \in A^{\prime} \cup T$ and $y \in A^{\prime} q \cup A^{\prime} r$, then $x \equiv y\left(\Theta^{\prime}\{\cdot\}+\Phi\right)$ iff $x \equiv$ $d\left(\Theta^{\prime}\{\cdot\}\right.$ and $b r \equiv y\left(\Theta^{\prime}\{\cdot\}+\Phi\right)$.

(i) of the claim is obvious. (ii)-(vii) are routine. We will prove (ii) as an example.

Let $x, y \in A^{\prime} \cup T$ and $\Theta \in H$. If $a \equiv b \equiv c \equiv 0(\Theta)$, then by (i), $\Theta^{\prime}\{\cdot\}+\Phi=\Theta^{\prime}\{\cdot\}$, and so $\langle x, y\rangle \in \Theta^{\prime}\{\cdot\}+\Phi$ iff $\langle x, y\rangle \in \Theta^{\prime}\{\cdot\}$. So we suppose it is not the case that $a \equiv b \equiv c \equiv 0(\Theta)$ and $x \equiv y\left(\Theta^{\prime}(\cdot)+\Phi\right)$. Then there exists $x=s_{0}, \cdots, s_{n}=y$ such that $s_{i} \equiv s_{i+1}$ under either $\Theta^{\prime}\{\cdot\}$ or $\Phi$. Let us suppose $n$ is minimal. So $s_{i} \neq s_{j}$ if $i \neq j$. Moreover, $s_{i} \equiv s_{i+1}\left(\Theta^{\prime}\{\cdot\}\right)$ iff $s_{i} \not \equiv s_{i+1}(\Phi)$ iff $s_{i+1} \equiv s_{i+2}(\Phi)$. Suppose $n>1$. Choose $l$ minimal so that $s_{l} \equiv s_{l+1}(\Phi)$. So $l=0$ or 1 . We have $x=s_{0} \equiv s_{l}\left(\Theta^{\prime}\{\cdot\}\right)$. By the assumption about $\Theta, u \equiv v\left(\Theta^{\prime}\{\cdot\}\right)$ and $v \in A^{\prime} p$ imply $u \in A^{\prime} p$. Similarly for $A^{\prime} q$ and $A^{\prime} r$. Since $x=s_{0} \notin A^{\prime} p \cup A^{\prime} q \cup$ $A^{\prime} r$, we have $s_{l} \in A^{\prime} \cup T$. Since $\left.\Phi\right|_{A^{\prime} \cup T}=\Delta$, we have $s_{l+1} \notin A^{\prime} \cup T$. Now $s_{l} \Phi s_{l+1}$ implies $\left\langle s_{l}, s_{l+1}\right\rangle=\langle c, a p\rangle$ or $\left\langle s_{l}, s_{l+1}\right\rangle=\langle d, b r\rangle$.

Let us suppose $s_{l}=c . \quad s_{l+1} \neq y$ because $y \in A^{\prime} \cup T$. So $s_{l+2}$ exists, and $a p=s_{l+1} \equiv s_{l+2}\left(\Theta^{\prime}\{\cdot\}\right)$. By the above reasoning, $s_{l+2} \in A^{\prime} p$ and $s_{l+2} \neq y$ and $s_{l+3}$ exists and $s_{l+2} \Phi s_{l+3}$. Since $s_{l+1} \neq s_{l+2} \neq s_{l+3}$, the definition of $\Phi$ yields $s_{l+2}=b p$ and $s_{l+3}=b q$. (Here we are using strongly the fact that $a \neq b$.) Continuing in this way we obtain $s_{l+7}=d$ and $n \geqq 7$. Recall $s_{l}=c$. We have $a p=s_{l+1} \equiv s_{l+2}=$ $b p\left(\Theta^{\prime}\{\cdot\}\right)$. Hence $\Theta_{\left.H^{\prime} \cdot \cdot\right\}}(a, b)=\left[\Theta_{\left.H^{\prime}\right\} \cdot \mid}(a, b) \wedge \Theta_{H^{\prime} \cdot \cdot \mid}(p, 0)\right]=\Theta_{\left.H^{\prime} \cdot \cdot\right\}}(a p, b p) \subseteq$ $\Theta^{\prime}\{\cdot\}$. Thus $\Theta_{H}(a, b) \leqq \Theta$ and $c \equiv d(\Theta)$. Thus, $s_{l} \equiv s_{l+7}\left(\Theta^{\prime}\{\cdot\}\right)$, and $n$ is not minimal. This is a contradiction. The case $s_{l}=d$ is similar. 
So $n=1$. As in the proof of Lemma 3.8, we have $x \equiv y\left(\Theta^{\prime}\{\cdot\}\right)$.

(iii)-(vii) of the claim are left to the reader.

It clearly follows from (ii)-(vii) of Claim 1 and Lemma 2.2 that $\left\{\theta^{\prime}\{\cdot\}+\Phi: \Theta \in H\right\}$ is a basis consisting of congruence relations of $\boldsymbol{A}^{\prime}[\cdot]$. By Lemma 2.4, $H_{\lambda}=\left\{\left(\Theta^{\prime}\{\cdot\}+\Phi\right) / \Phi: \Theta \in H\right\}$ is a basis consisting of congruence relations of $\boldsymbol{A}_{\lambda}$.

It is clear that (i)-(v) of the extension definition and (A)-(D) of (\#) hold for $\left\langle\boldsymbol{A}_{\lambda}, H_{\lambda}, I_{\lambda}, O_{\lambda}\right\rangle$ vis-à-vis $\left\langle\boldsymbol{A}^{\prime}, H^{\prime}, I^{\prime}, O^{\prime}\right\rangle$.

For any $a, b \in A^{\prime}$ we have, in $A^{\prime}\{\cdot\},\langle a 0, b 0\rangle \in \bigcap\left(H^{\prime}\{\cdot\}\right)=(\bigcap H)^{\prime}\{\cdot\} \cong$ $\left((\cap H)^{\prime}\{\cdot\}\right)+\Phi$. So in $\boldsymbol{A}_{\lambda}$, we have, for any $a, b \in A^{\prime}$, that $\langle a 0, b 0\rangle \in$ $\left(\left((\cap H)^{\prime}\{\cdot\}\right)+\Phi\right) / \Phi=\bigcap H_{\lambda}$. Thus (E) of (\#) holds for $A_{\lambda}$ and $H_{\lambda}$.

For every $x \in A^{\prime} \cup T$ there is an $e \in A^{\prime}$ satisfying $e$ CLS $x$ (in $A^{\prime}$, $\left.\bmod H^{\prime}\{\cdot\}\right)$. By (ii) of Claim 1, we have, given the same $x$ and $e$, $e \operatorname{CLS} x$ (in $\left.A^{\prime}, \bmod H_{2}\right)$.

Recall, in what follows, that closest things need not be unique. Also, it may appear to the reader that there is some apparent ambiguity as to $\Theta_{H_{\lambda}}(x, y)$ as given by (ii)-(vii) of Claim 1. Lemma 2.4 assures us that this apparent ambiguity is not real.

(vi) of Claim 1 implies that $c$ CLS $x$ (in $A^{\prime}, \bmod H_{\lambda}$ ) holds for any $x \in A^{\prime} p \cup A^{\prime} q$. (vii) yields that $d$ CLS $x$ (in $A^{\prime}$, $\bmod H_{2}$ ) holds for any $x \in A^{\prime} q \cup A^{\prime} r$.

We have established (F) of (\#) for $A_{\lambda}$ and $H_{\lambda}$.

Certainly $A_{\lambda}^{2}=I_{\lambda} \cup O_{\lambda}$. Suppose $\langle x, y\rangle \in O_{\lambda}$. Then one of the following holds:

(i) $\langle x, y\rangle=\langle u / \Phi, v / \Phi\rangle$ and $\langle u, v\rangle \in O^{\prime}\{\cdot\}$ and $u, v \in A^{\prime} \cup T$;

(ii) $\langle x, c\rangle$ satisfies (i) and $y \in A^{\prime} p \cup A^{\prime} q$;

(iii) $\langle x, d\rangle$ satisfies (i) and $y \in A^{\prime} q \cup A^{\prime} r$;

(iv) $\langle c, d\rangle \in O$ and $x \in A^{\prime} p$ and $y \in A^{\prime} r$;

( v) $\langle y, x\rangle$ satisfies one of (ii)-(iv).

Claim 1 makes it obvious, that in each of these cases, $x \equiv$ $0\left(\Theta_{H_{\lambda}}(x, y)\right)$. We have established (i) of (G) of (\#) for $O_{\lambda}$ and $H_{\lambda}$. of $(\mathrm{G})$ of $(\#)$ for $I_{\lambda}$ is obvious.

We know $I^{\prime}[\cdot]$ and $H^{\prime}\{\cdot\}$ satisfy (iii) of (G) of (\#). Recall our description of $H_{\lambda}$-closest things in $A^{\prime}$ and that $\langle c, c\rangle$ and $\langle d, d\rangle$ are in $I \subseteq I_{\lambda}$. Now the definition of $I_{\lambda}$ makes it plain that (iii) of (G) of (\#) holds for $H_{\lambda}$ and $I_{\lambda}$.

Suppose $e, f, g, h \in A^{\prime}$ and $\langle f, h\rangle \in I_{\lambda}$. Then $\langle f, h\rangle \in I^{\prime}$.

If $\langle f, h\rangle \in\{\langle p, p\rangle,\langle q, q\rangle,\langle r, r\rangle\}$, then $\langle e f, g h\rangle \in I_{\lambda}$ by (ii) of its definition. Otherwise, $\langle f, h\rangle \in I$ and $\langle e f, g h\rangle \in A^{\prime} \cup T$. Whence, $\langle e f, g h\rangle \in I^{\prime}\{\cdot\}$ and (i) of the definition of $I_{\lambda}$ is satisfied. So (a) of (iv) of (G) of (\#) holds for $\boldsymbol{A}_{\lambda}$ and $H_{\lambda}$.

If $\langle f, h\rangle \in\{\langle p, p\rangle,\langle q, q\rangle,\langle r, r\rangle\}$, then (iii) of Claim 1 and (\#) for $\left\langle\boldsymbol{A}^{\prime}[\cdot], H^{\prime}\{\cdot\}, \cdots\right\rangle$ imply that $\Theta_{H_{i}}(e f, g h)=\left(\left(\Theta_{H^{\prime} \cdot \cdot \cdot(}(e f, g h)\right)+\Phi\right) / \Phi=$ $\left(\left(\Theta_{H^{\prime}\{\cdot\}}(e, g) \wedge \Theta_{\left.H^{\prime} \cdot \cdot\right\}}(f, 0)\right)+\Phi\right) / \Phi$, since $f=h,=\left(\left(\left(\Theta_{\left.H^{\prime} \cdot \cdot\right\}}(e, g)\right)+\Phi\right) \wedge\right.$ 
$\left.\Theta_{\left.H^{\prime} \cdot \cdot\right\}}(f, o)\right) / \Phi$, since $\Phi \subseteq \Theta_{\left.H^{\prime} \mid \cdot\right\}}(f, 0)$ and since $\Theta \rightarrow(\Theta+\Phi)$ is an order isomorphism between the bases $H^{\prime}\{\cdot\}$ and $\left\{\Theta^{\prime}\{\cdot\}+\Phi: \Theta \in H\right\}$, = $\left(\left(\Theta_{H^{\prime}|\cdot|}(e, g)\right)+\Phi\right) / \Phi \wedge \Theta_{\left.H^{\prime} \mid \cdot\right\}}(f, 0) / \Phi=\Theta_{H_{\lambda}}(e, g) \wedge \Theta_{H_{\lambda}}(f, 0)=\left[\Theta_{H_{\lambda}}(e, g) \wedge\right.$ $\left.\Theta_{H_{\lambda}}(f, 0)\right] \vee \Theta_{H_{\lambda}}(f, h)$ since $f=h$. So we may suppose $\langle f, h\rangle \in I$. Then $e f, g h \in A^{\prime} \cup T$. Then (ii) of Claim 1 and a similar calculation yield the desired result. That is, (b) of (iv) of (G) of (\#) holds for $\boldsymbol{A}_{\lambda}$ and $H_{\lambda}$.

(i) and (ii) of the definition of $I_{\lambda}$ yield (v) of (G) of ( $\$$ ) for $I_{\lambda}$ and $\boldsymbol{A}_{\lambda}$. Now we apply Lemma 1 and transitivity to obtain (ii) of the lemma.

The proof of (iii) of the lemma is just like the proof of Lemma 3.8.(iii), and it is obvious anyway.

5. Sums and products. In this section we make a few, previously known and simple observations about congruence relations on direct products and direct sums of algebras.

Suppose $\left(\boldsymbol{A}_{i}: i \in I\right)$ is a family of pointed algebras. $\Pi \boldsymbol{A}_{i}$ and $\Pi\left(\boldsymbol{A}_{i}: i \in I\right)$ will denote the direct product of this family. $\Sigma A_{i}$ and $\Sigma\left(A_{i}: i \in I\right)$ will denote $\left\{x \in \prod A_{i}:\{i \in I: x(i) \neq 0\}\right.$ is finite $\} . \Sigma A_{i}$ and $\Sigma\left(A_{i}: i \in I\right)$ will denote the corresponding algebra.

Suppose $\left(\boldsymbol{A}_{i}: i \in I\right)$ is a family of algebras and $\Theta_{i} \in \operatorname{Con} \boldsymbol{A}_{i}$ for each $i$. For $x, y \in \Pi A_{i}$ we let $x \Pi \Theta_{i} y$ (or $x \equiv y\left(\Pi \Theta_{i}\right)$ ) iff $x \Theta_{i} y$ for all $i \in I$. If the $\boldsymbol{A}_{i}$ 's are pointed algebras, we will also use $\Pi \Theta_{i}$ to denote $\left.\Pi \Theta_{i}\right|_{A_{i}}$. A congruence is rectangular iff it is of the form $\Pi \Theta_{i}$.

By studying when a pair is in a join of congruence relations we easily see

Fact 1. (i) If $I$ is finite, then in $\operatorname{Con}\left(\Pi \boldsymbol{A}_{i}\right)$ the join of rectangular congruences is rectangular.

(ii) If each $\boldsymbol{A}_{i}$ is a pointed algebra, then in $\operatorname{Con}\left(\Sigma \boldsymbol{A}_{i}\right)$ the join of rectangular congruences is rectangular.

CoROLLARY 1. The mapping $\left\langle\Theta_{i}: i \in I\right\rangle \rightarrow \Pi \Theta_{i}$ embeds $\Pi\left(\operatorname{Con}\left(\boldsymbol{A}_{i}\right)\right.$ : $i \in I)$ into $\operatorname{Con}\left(\Pi \boldsymbol{A}_{i}\right)$, if $I$ is finite, and into $\operatorname{Con}\left(\Sigma \boldsymbol{A}_{i}\right)$, if each $\boldsymbol{A}_{i}$ is a pointed algebra. obtain

Since every congruence is a join of principal congruences, we

Corollary 2. (i) Suppose I is finite. If each principal congruence of $\Pi \boldsymbol{A}_{i}$ is rectangular, then every congruence of $\Pi \boldsymbol{A}_{i}$ is rectangular, and $\operatorname{Con}\left(\Pi \boldsymbol{A}_{i}\right)$ is isomorphic to $\Pi \operatorname{Con}\left(\boldsymbol{A}_{i}\right)$.

(ii) Suppose each $\boldsymbol{A}_{i}$ is a pointed algebra. If each principal 
congruence of $\Sigma \boldsymbol{A}_{i}$ is rectangular, then every congruence relation of $\Sigma \boldsymbol{A}_{i}$ is rectangular, and $\operatorname{Con}\left(\Sigma \boldsymbol{A}_{i}\right)$ is isomorphic to $\Pi \operatorname{Con}\left(\boldsymbol{A}_{i}\right)$.

Definition. For $i<4$, let $t_{i}$ be the 4-ary term $x_{0} \cdot x_{i}$. Let $\tau=$ $\left\langle t_{1}, t_{2}, t_{3}\right\rangle . \quad \tau$ defines the principal congruences of $\boldsymbol{A}$ if and only if for every $a, b, c, d \in A$, it holds that $\langle c, d\rangle \in \Theta(a, b)$ iff there exist $p, q, r \in A$ with $c=t_{1}(a, p, q, r)$ and $t_{1}(b, p, q, r)=t_{2}(b, p, q, r)$ and $t_{2}(a, p, q, r)=t_{3}(a, p, q, r)$ and $t_{3}(b, p, q, r)=d$.

Let $a, b \in \Pi A_{i}$, assuming definability by $\tau$, one can easily show $\Pi \Theta\left(a_{i}, b_{i}\right) \leqq \Theta(a, b)$. The proof works equally well in $\Pi \boldsymbol{A}_{i}$ and in $\Sigma \boldsymbol{A}_{i}$. This yields

Fact 2. Suppose $\tau$ defines the principal congruences of each $\boldsymbol{A}_{i}$.

(i) Every principal congruence of $\Pi \boldsymbol{A}_{i}$ is rectangular.

(ii) If each $\boldsymbol{A}_{i}$ is a pointed algebra, then every principal congruence of $\Sigma \boldsymbol{A}_{i}$ is rectangular.

Obviously, we have

THEOREM 4. Suppose $\tau$ defines the principal congruences of each $\boldsymbol{A}_{i}$.

(i) If $I$ is finite, then $\operatorname{Con}\left(\Pi \boldsymbol{A}_{i}\right)$ is isomorphic to $\Pi \operatorname{Con}\left(\boldsymbol{A}_{i}\right)$.

(ii) If each $\boldsymbol{A}_{i}$ is a pointed algebra, then $\operatorname{Con}\left(\Sigma \boldsymbol{A}_{i}\right)$ is isomorphic to $\Pi \operatorname{Con}\left(\boldsymbol{A}_{i}\right)$.

Obviously, a much more general theorem can be obtained. In particular, in any variety having Uniform Congruence Schemes the congruences are "productive." Fried, Grätzer and Quackenbush observed, essentially, this in the trivial halves of Theorems 3.5 and 5.2 of $[6]$.

6. Final remarks. Clearly Theorem 1 is an immediate consequence of Theorems 2, 3, and 4 .

The representation provided in Theorem 1 is type-3. If we also supposed $L$ is modular, could we then have produced a type-2 representation in the proof of Theorem 1? Most likely, that is the case. Ideas as to how this might be done can be gleaned from Appendix 7 of the 2nd edition of [8].

There is an asymmetry vis-à-vis - in the representation provided in the proof of Theorem 3. Do there exist distributive algebraic lattices which can be represented as congruence lattices of groupoids but which have no representation as a congruence lattice of a commutative groupoid?

In [25] Walter Taylor provides a countable algebraic lattice that 
is not the congruence lattice of any semigroup. One of course wonders if there is any countable algebraic lattice that is not the congruence lattice of a groupoid. It may even be the case that there is some algebra $A$ of type $\langle 2,1\rangle$ such that Con $A$ is not isomorphic to the congruence lattice of any groupoid.

It can also be shown that Taylor's example is not the congruence lattice of any unary algebra having only finitely many operations. This we now proceed to do. We suppose the reader has some familiarity with Taylor's example.

$C$ is to be the semilattice of compact elements.

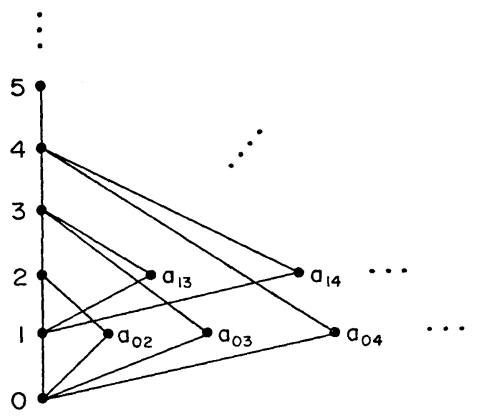

$C$ consists of the chain $\omega(0<1<2<3<\cdots)$ together with elements $a_{i j}(0 \leqq i<j-1)$ with $i<a_{i j}<j$. Now we let $L$ be the ideal lattice of $C$.

THeOREM 5. If $L$ is isomorphic to Con $\boldsymbol{A}$ and $\boldsymbol{A}$ is a unary algebra, then $\boldsymbol{A}$ has infinitely many operations.

Proof. Suppose on the contrary that $L \cong \operatorname{Con} A$ and $\boldsymbol{A}$ is a unary algebra having only finitely many operations. Note that in $C$, or in $L$, the filter (dual ideal) generated by $n,[n)$, is isomorphic to $C$, or $L$, as the case may be.

Thus, we may assume there is an element $0 \in A$ such that $f(0)=0$ for each of the finitely many operations of $\boldsymbol{A}$. That is, we may assume $\boldsymbol{A}$ is a pointed algebra.

By an abuse of notation, we will refer to the congruences of $\boldsymbol{A}$ by their preimages under the isomorphism, namely $0,1, a_{02}, a_{03}, a_{04}$, $\cdots, 2, a_{13}, \cdots$ etc. (Note 0 names both an element of $A$ and a congruence of $\boldsymbol{A}$. Context should make clear which is which.)

We may also assume, using the above fact about $n \in \omega$, that $1=\Theta(0, x)$ for some $x \in A$. We see that $\langle 0, x\rangle \in a_{02} \vee a_{03}$ and $\langle 0, x\rangle \in a_{04} \vee a_{05}$. So we have sequences $0=s_{0}, s_{1}, \cdots, s_{k}=x$ and $0=r_{0}, r_{1}, \cdots, r_{m}=x$ with $s_{i} \equiv s_{i+1}$ under $a_{02}$ or $a_{03}$ and $r_{i} \equiv r_{i+1}$ under $a_{04}$ or $a_{05}$, for each possible $i$. We may suppose $s_{0} \neq s_{1}$ and $r_{0} \neq r_{1}$. Let $u=s_{1}$ and $v=r_{1}$. We have $\Theta(0, u)=a_{02}$ or $a_{03}$ and $\Theta(0, v)=a_{04}$ or $a_{05}$. 
Case 1. $\Theta(0, u)=a_{02}$ and $\Theta(0, v)=a_{04} \cdot \theta(u, v)$ is compact, and thus, in $C$. Also $a_{04}=\Theta(0, v) \subseteq \Theta(0, u) \vee \Theta(u, v)=a_{02} \vee \Theta(u, v)$, and $a_{02}=\Theta(0, u) \subseteq \Theta(0, v) \vee \Theta(u, v)=a_{04} \vee \Theta(u, v)$, and $\Theta(u, v) \subseteq \Theta(0, u) \vee$ $\Theta(0, v)=a_{02} \vee a_{04}$. By inspecting $C$, we see that $\Theta(u, v)$ is either 4 or $a_{14}$ or $a_{24}$. In any case $\Theta(u, v) \geqq 1=\Theta(0, x)$.

Since $\boldsymbol{A}$ is a unary algebra, every unary algebraic function of $\boldsymbol{A}$ is a unary term (unary polynomial) or a constant function. For any unary term $t$, we obviously have $t(0)=0$. Now, since $\langle 0, x\rangle \in$ $\Theta(u, v)$, there are unary terms $t_{1}, \cdots, t_{l}$ such that $0 \in\left\{t_{1}(u), t_{1}(v)\right\}$ and for $1 \leqq i<l\left\{t_{i}(u), t_{i}(v)\right\} \cap\left\{t_{i+1}(u), t_{i+1}(v)\right\} \neq \varnothing$ and $x \in\left\{t_{l}(u), t_{l}(v)\right\}$. Since $t_{l}$ is a term, $t_{l}(0)=0$. Since $x=t_{l}(u)$ or $x=t_{l}(v)$, we have $\langle 0, x\rangle \in \Theta(0, u)$ or $\langle 0, x\rangle \in \Theta(0, v)$. So $1=\Theta(0, x) \subseteq \Theta(0, u)=a_{02}$ or $1=\Theta(0, x) \subseteq \Theta(0, v)=a_{0 !}$. But $1, a_{02}, a_{04}$ are distinct atoms.

The three remaining cases yield similar contradictions. So the initial contrary assumption is false. This ends the proof.

Consider Mal'tsev's lemma (Theorem 3, p. 54, [8]). The above proof actually shows that whenever $L \cong \operatorname{Con} A$, the sequences of unary algebraic functions " $p_{0}, \cdots, p_{n-1}$ " cannot all be sequences of unary terms (term functions). In other words, the above provides some "technical specifications" for any successful representation of $L$ as $\operatorname{Con} \boldsymbol{A}$, where $\boldsymbol{A}$ is of finite type, even if $\boldsymbol{A}$ is nonunary.

Clearly, all of the above is true for a very "narrow" sublattice of $L$. Condition $\left(^{*}\right)$ (see Part I) and Lemma 1 of Part I did not enter into the proof.

Ralph Freese has shown that this same $L$ is not the congruence lattice of any groupoid possessing a two-sided identity element.

The conclusion of Lemma 1 of Part $I$ is now called the term condition or T. C. $(1,1,0)$. This condition has become quite important in a context quite unrelated to Part I. R. McKenzie coined the term after first seeing Lemma 1 . The condition first appeared in Theorem 9 of H. Werner's paper [26]. McKenzie employed this condition in [18]. My first exposure to it occurred when McKenzie's paper was presented in our Hawaii seminar in 1976. This was six months before I proved Lemma 1 . I had forgotten about the contents of [18] till Taylor's paper [25] reminded me in 1979. (This explains why this paragraph is in Part II instead of Part I, where it belongs.)

Although it is a fairly well-known theorem, in [17] I gave only some corollaries of the following

Folklore Theorem. If $L$ is a distributive, algebraic, and dually algebraic lattice, then $L$ is isomorphic to the congruence lattice of some groupoid. 
Proof. Such an $L$ is isomorphic to the lattice of nonempty hereditary subsets of some partially ordered set $P$ having a least element 0 . Let $A=\langle P, \cdot\rangle$ where $x \cdot y=y$ if $y \leqq x$ and $x \cdot y=0$ otherwise. Each congruence has at most one nontrivial class and this class is a hereditary subset of $P$. The required isomorphism is obvious.

By generalizing Fact 2 of $\S 5$ appropriately, one can now show easily that if $L$ is isomorphic to the product of a family of lattices each of which is either a pinched lattice, or the ideals of a distributive lattice, or a distributive, algebraic and dually algebraic lattice, then $L$ can be represented as the congruence lattice of a groupoid. There is still a lot to be done. This does not even exhaust the class of distributive algebraic lattices.

We remind the reader of the problems listed in Part I (see [5]).

\section{REFERENCES}

1. H. Andréka and I. Németi, Similarity types, pseudosimple algebras and congruence representation of chains, Algebra Universalis, 13 (1981), 293-306.

2. P. Crawley and R. P. Dilworth, Algebraic Theory of Lattices, Prentice-Hall, Englewood Cliffs, N. J., 1973.

3. G. Fraser and A. Horn, Congruence relations in direct products, Proc. Amer. Math. Soc., 26 (1970), 390-394.

4. R. Freese, Congruence lattices of finitely generated modular lattices, Proc. Lattice Theory Conf., Ulm 1975, G. Kalmbach, Editor, 62-70.

5. R. Freese, W. A. Lampe, and W. Taylor, Congruence lattices of algebras of fixed similarity type, I. Pacific J. Math., 82 (1979), 59-68.

6. E. Fried, G. Grätzer, and R. Quackenbush, Uniform congruence schemes, Algebra Universilis, 10 (1980), 176-188.

7. - The equational class generated by weakly associative lattices with the unique bound property, 1978 manuscript.

8. G. Grätzer, Universal Algebra, D. Van Nostrand Co., Inc. Princeton, N. J., 1968, or Springer-Verlag New York Inc., New York, N. Y., 1979.

9. L Lattice Theory, First Concepts and Distributive Lattices, H. M. Freeman, San Francisco, 1971.

10. G. Grätzer and E. T. Schmidt, On congruences of lattices, Acta Math. Acad. Sci. Hungar., 13 (1962), 179-185.

11. Characterizations of congruence lattices of abstract algebras, Acta Sci. Math. (Szeged), 24 (1963), 34-59.

12. W. Hanf, Representations of lattices of subalgebras (Preliminary report), Bull. Amer. Math. Soc., 62 (1956), 402.

13. B. Jónsson, Topics in Universal Algebra, Lecture Notes in Mathematics, vol. 250, Springer-Verlag, Berlin, 1972.

14. — Varieties of algebras and their congruence varieties, Proc. Int. Congress Math., Vancouver, (1974), 315-320.

15. W. A. Lampe, The independence of certain related structures of a universal algebra I-IV, Algebra Universalis, 2 (1972), 99-112, 270-283, 286-295, 296-302.

16. - Notes on the congruence lattices of algebras of finite type, 1975 manuscript.

17. Congruence lattice representations and similarity type, Colloquiua Mathe- 
matica Societatis János Bolyai, 29. Universal Algebra, Esztergom, (Hungary) 1977, North Holland 1980, 495-500.

18. R. McKenzie, On minimal, locally finite varieties with permuting congruence relations, 1976 manuscript.

19. - Para primal varieties: a study of finite axiomatizability and definable principal congruences in locally finite varieties, Algebra Universalis, 8 (1978), 336-348. 20. P. Pudlák, $A$ new proof of the congruence lattice representation theorem, Algebra Universalis, 6 (1976), 269-275.

21. E. T. Schmidt, Kongruenzrelationen algebraischer Strukturen, Mathematische Forschungsberichte, VEB Deutscher Verlag, Berlin, 1969, MR 47 \#136.

22. - Every finite distributive lattice is the congruence lattice of a modular lattice, Algebra Universalis, 4 (1974), 49-57.

23. - Congruence lattices of complemented modular lattices, Colloquia Mathematica Societatis János Bolyai, 29. Universal Algebra, Esztergom, (Hungary) 1977, North Holland 1980.

24. - The ideal lattice of a distributive lattice with 0 is the congruence lattice of a lattice, 1980 manuscript.

25. W. Taylor, Some applications of the term condition, Algebra Universalis, 14 (1982), 11-24.

26. H. Werner, Congruences on products of algebras, Algebra Universalis, 4 (1974), 99-105.

Received January 14, 1981.

UNIVERSITY OF HAWAII

HoNOLULU, HI 96822 


\title{
PACIFIC JOURNAL OF MATHEMATICS
}

\section{EDITORS}

\author{
DONALD BABBITT (Managing Editor) \\ University of California \\ Los Angeles, CA 90024 \\ Hugo Rossi \\ University of Utah \\ Salt Lake City, UT 84112 \\ C. C. MOORE and ARthur Agus \\ University of California \\ Berkeley, CA 94720
}

J. DUGUNDJI

Department of Mathematics

University of Southern California

Los Angeles, CA 90007

R. FINN and J. MILGRAM

Stanford University

Stanford, CA 94305

\section{ASSOCIATE EDITORS}
R. ARENS
E. F. BeCKENBACH
B. H. NeumanN
F. WoLF
K. YoSHIDA

\section{SUPPORTING INSTITUTIONS}

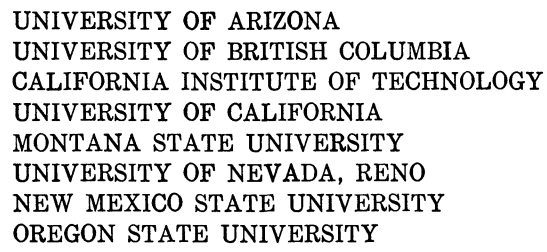

UNIVERSITY OF ARIZONA

UNIVERSITY OF BRITISH COLUMBIA

CALIFORNIA INSTITUTE OF TECHNOLOGY

UNIVERSITY OF CALIFORNIA

MONTANA STATE UNIVERSITY

UNIVERSITY OF NEVADA, RENO

NEW MEXICO STATE UNIVERSITY

OREGON STATE UNIVERSITY

\author{
UNIVERSITY OF OREGON \\ UNIVERSITY OF SOUTHERN CALIFORNIA \\ STANFORD UNIVERSITY \\ UNIVERSITY OF AAWAII \\ UNIVERSITY OF TOKYO \\ UNIVERSITY OF UTAH \\ WASHINGTON STATE UNIVERSITY \\ UNIVERSITY OF WASHINGTON
}

The Supporting Institutions listed above contribute to the cost of publication of this Journal, but they are not owners or publishers and have no responsibility for its content or policies,

Mathematical parers intended for publication in the Pacific Journal of Mathematics should be in typed form or offset-reproduced, (not dittoed), double spaced with large margins. Please do not use built up fractions in the text of the manuscript. However, you may use them in the displayed equations. Underline Greek letters in red, German in green, and script in blue. The first paragraph or two must be capable of being used separately as a synopsis of the entire paper. Please propose a heading for the odd unmbered pages of less than 35 characters. Manuscripts, in triplicate, may be sent to any one of the editors. Please classify according to the scheme of Math. Reviews, Index to Vol. 39. Supply name and address of author to whom proofs should be sent. All other communications should be addressed to the managing editor, or Elaine Barth, University of California, Los Angeles, California, 90024.

50 reprints to each author are provided free for each article, only if page charges have been substantially paid. Additional copies may be obtained at cost in multiples of 50 .

The Pacific Journal of Mathematics is issued monthly as of January 1966, Regular subscription rate: $\$ 114.00$ a year (6 Vol., 12 issues). Special rate: $\$ 57.00$ a year to individual members of supporting institution.

Subscriptions, orders for numbers issued in the last three calendar years, and changes of address shoud be sent to Pacific Journal of Mathematics, P.O. Box 969, Carmel Valley, CA 93924, U.S.A. Old back numbers obtainable from Kraus Periodicals Co., Route 100, Millwood, NY 10546.

PUBLISHED BY PACIFIC JOURNAL OF MATHEMATICS, A NON-PROFIT CORPORATION

Printed at Kokusai Bunken Insatsusha (International Academic Printing Co., Ltd.). 8-8, 3-chome, Takadanobaba, Shinjuku-ku, Tokyo 160, Japan. 


\section{Pacific Journal of Mathematics}

\section{Vol. 103, No. $2 \quad$ April, 1982}

Alberto Alesina and Leonede De Michele, A dichotomy for a class of positive

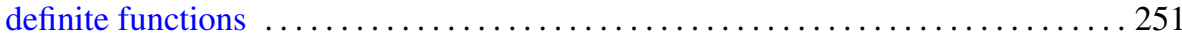

Kahtan Alzubaidy, Rank 2 -groups, $p>3$, and Chern classes . . . . . . . . . . 259

James Arney and Edward A. Bender, Random mappings with constraints on

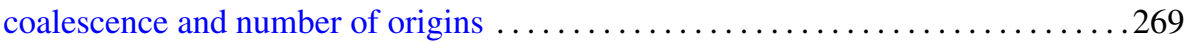

Bruce C. Berndt, An arithmetic Poisson formula . . . . . . . . . . . . . . . 295

Julius Rubin Blum and J. I. Reich, Pointwise ergodic theorems in 1.c.a. groups . . . 301

Jonathan Borwein, A note on $\varepsilon$-subgradients and maximal monotonicity . . . . . . . 307

Andrew Michael Brunner, Edward James Mayland, Jr. and Jonathan Simon,

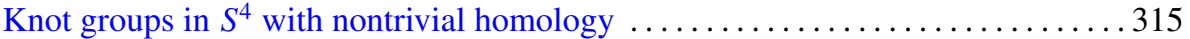

Luis A. Caffarelli, Avner Friedman and Alessandro Torelli, The two-obstacle

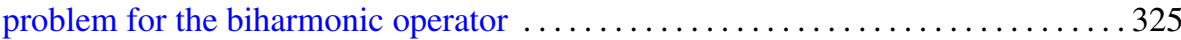

Aleksander Całka, On local isometries of finitely compact metric spaces . . . . . . 337

William S. Cohn, Carleson measures for functions orthogonal to invariant

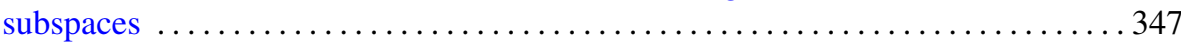

Roger Fenn and Denis Karmen Sjerve, Duality and cohomology for one-relator

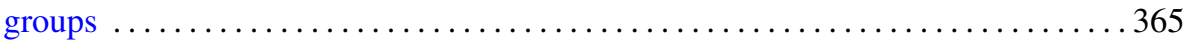

Gen Hua Shi, On the least number of fixed points for infinite complexes . . . . . . . 377

George Golightly, Shadow and inverse-shadow inner products for a class of linear

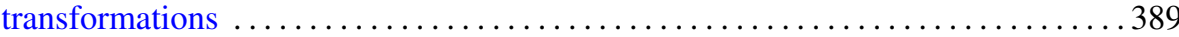

Joachim Georg Hartung, An extension of Sion's minimax theorem with an

application to a method for constrained games $\ldots \ldots \ldots \ldots \ldots \ldots \ldots \ldots \ldots \ldots . \ldots 4$

Vikram Jha and Michael Joseph Kallaher, On the Lorimer-Rahilly and

Johnson-Walker translation planes

Kenneth Richard Johnson, Unitary analogs of generalized Ramanujan sums .

Peter Dexter Johnson, Jr. and R. N. Mohapatra, Best possible results in a class of inequalities

Dieter Jungnickel and Sharad S. Sane, On extensions of nets

Johan Henricus Bernardus Kemperman and Morris Skibinsky, On the

characterization of an interesting property of the arcsin distribution ...

Karl Andrew Kosler, On hereditary rings and Noetherian $V$-rings

William A. Lampe, Congruence lattices of algebras of fixed similarity type. II . . . . 475

M. N. Mishra, N. N. Nayak and Swadeenananda Pattanayak, Strong result for real zeros of random polynomials

Sidney Allen Morris and Peter Robert Nickolas, Locally invariant topologies on free groups

Richard Cole Penney, A Fourier transform theorem on nilmanifolds and nil-theta functions

Andrei Shkalikov, Estimates of meromorphic functions and summability theorems

László Székelyhidi, Note on exponential polynomials

William Thomas Watkins, Homeomorphic classification of certain inverse limit spaces with open bonding maps $\ldots \ldots \ldots \ldots \ldots \ldots \ldots \ldots$

David G. Wright, Countable decompositions of $E^{n}$

Takayuki Kawada, Correction to: "Sample functions of Pólya processes" .

Z. A. Chanturia, Errata: "On the absolute convergence of Fourier series of the 